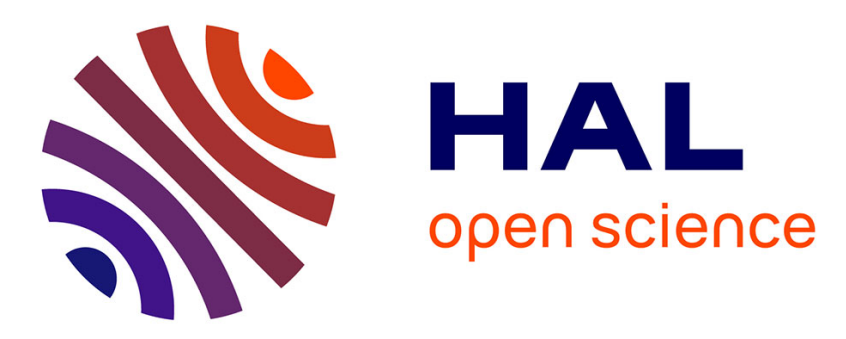

\title{
A Parallel Spitting Method for Coupled Monotone Inclusions
}

Hedy Attouch, Luis M. Briceno-Arias, Patrick Louis Combettes

\section{To cite this version:}

Hedy Attouch, Luis M. Briceno-Arias, Patrick Louis Combettes. A Parallel Spitting Method for Coupled Monotone Inclusions. SIAM Journal on Control and Optimization, 2010, 48 (5), pp.32463270. 10.1137/090754297 . hal-00803883

\section{HAL Id: hal-00803883 https://hal.science/hal-00803883}

Submitted on 24 Mar 2013

HAL is a multi-disciplinary open access archive for the deposit and dissemination of scientific research documents, whether they are published or not. The documents may come from teaching and research institutions in France or abroad, or from public or private research centers.
L'archive ouverte pluridisciplinaire HAL, est destinée au dépôt et à la diffusion de documents scientifiques de niveau recherche, publiés ou non, émanant des établissements d'enseignement et de recherche français ou étrangers, des laboratoires publics ou privés. 


\title{
A PARALLEL SPLITTING METHOD FOR COUPLED MONOTONE INCLUSIONS*
}

\author{
Hédy Attouch, ${ }^{1}$ Luis M. Briceño-Arias, ${ }^{2}$ and Patrick L. Combettes ${ }^{3}$ \\ ${ }^{1}$ Université Montpellier II \\ Institut de Mathématiques et de Modélisation de Montpellier - UMR 5149 \\ 34095 Montpellier Cedex 5, France (attouch@math.univ-montp2.fr) \\ ${ }^{2}$ UPMC Université Paris 06 \\ Laboratoire Jacques-Louis Lions - UMR 7598 and Équipe Combinatoire et Optimisation - UMR 7090 \\ 75005 Paris, France (lbriceno@math.jussieu.fr) \\ ${ }^{3}$ UPMC Université Paris 06 \\ Laboratoire Jacques-Louis Lions - UMR 7598 and 75005 Paris, France (plc@math.jussieu.fr) \\ September 29, 2009 -- version 1.27

\begin{abstract}
A parallel splitting method is proposed for solving systems of coupled monotone inclusions in Hilbert spaces and its convergence is established under the assumption that solutions exist. Unlike classical alternating algorithms, which are limited to two variables and linear coupling, our parallel method can handle an arbitrary number of variables as well as nonlinear coupling schemes. The breadth and flexibility of the proposed framework is illustrated through applications in the areas of evolution inclusions, variational problems, best approximation, and network flows.
\end{abstract}

Keywords: coupled systems, demiregular operator, evolution inclusion, forward-backward algorithm, maximal monotone operator, operator splitting, parallel algorithm, weak convergence.

\footnotetext{
${ }^{*}$ Contact author: P. L. Combettes, plc@math.jussieu.fr, phone: +3314427 6319, fax: +33 144277200 . This work was supported by the Agence Nationale de la Recherche under grant ANR-08-BLAN-0294-02.
} 


\section{Problem statement}

This paper is concerned with the numerical solution of systems of coupled monotone inclusions in Hilbert spaces. A simple instance of this problem is to

$$
\text { find } x_{1} \in \mathcal{H}, x_{2} \in \mathcal{H} \quad \text { such that }\left\{\begin{array}{l}
0 \in A_{1} x_{1}+x_{1}-x_{2} \\
0 \in A_{2} x_{2}+x_{2}-x_{1}
\end{array}\right.
$$

where $(\mathcal{H},\|\cdot\|)$ is a real Hilbert space, and where $A_{1}$ and $A_{2}$ are maximal monotone operators acting on $\mathcal{H}$. This formulation arises in various areas of nonlinear analysis [12]. For example, if $A_{1}=\partial f_{1}$ and $A_{2}=\partial f_{2}$ are the subdifferentials of proper lower semicontinuous convex functions $f_{1}$ and $f_{2}$ from $\mathcal{H}$ to $]-\infty,+\infty],(1.1)$ is equivalent to

$$
\underset{x_{1} \in \mathcal{H}, x_{2} \in \mathcal{H}}{\operatorname{minimize}} f_{1}\left(x_{1}\right)+f_{2}\left(x_{2}\right)+\frac{1}{2}\left\|x_{1}-x_{2}\right\|^{2} .
$$

This joint minimization problem, which was first investigated in [1], models problems in disciplines such as the cognitive sciences [4], image processing [26], and signal processing [28] (see also the references therein for further applications in mechanics, filter design, and dynamical games). In particular, if $f_{1}$ and $f_{2}$ are the indicator functions of closed convex subsets $C_{1}$ and $C_{2}$ of $\mathcal{H},(1.2)$ reverts to the classical best approximation pair problem $[8,11,18,29]$

$$
\underset{x_{1} \in C_{1}, x_{2} \in C_{2}}{\operatorname{minimize}}\left\|x_{1}-x_{2}\right\|
$$

On the numerical side, a simple algorithm is available to solve (1.1), namely,

$$
x_{1,0} \in \mathcal{H} \quad \text { and } \quad(\forall n \in \mathbb{N}) \quad\left\{\begin{array}{l}
x_{2, n}=\left(\mathrm{Id}+A_{2}\right)^{-1} x_{1, n} \\
x_{1, n+1}=\left(\mathrm{Id}+A_{1}\right)^{-1} x_{2, n} .
\end{array}\right.
$$

This alternating resolvent method produces sequences $\left(x_{1, n}\right)_{n \in \mathbb{N}}$ and $\left(x_{2, n}\right)_{n \in \mathbb{N}}$ that converge weakly to points $x_{1}$ and $x_{2}$, respectively, such that $\left(x_{1}, x_{2}\right)$ solves (1.1) if solutions exist [12, Theorem 3.3]. In [3], the variational formulation (1.2) was extended to

$$
\underset{x_{1} \in \mathcal{H}_{1}, x_{2} \in \mathcal{H}_{2}}{\operatorname{minimize}} f_{1}\left(x_{1}\right)+f_{2}\left(x_{2}\right)+\frac{1}{2}\left\|L_{1} x_{1}-L_{2} x_{2}\right\|_{\mathcal{G}}^{2},
$$

where $\mathcal{H}_{1}, \mathcal{H}_{2}$, and $\mathcal{G}$ are Hilbert spaces, $\left.\left.f_{1}: \mathcal{H}_{1} \rightarrow\right]-\infty,+\infty\right]$ and $\left.\left.f_{2}: \mathcal{H}_{2} \rightarrow\right]-\infty,+\infty\right]$ are proper lower semicontinuous convex functions, and $L_{1}: \mathcal{H}_{1} \rightarrow \mathcal{G}$ and $L_{2}: \mathcal{H}_{2} \rightarrow \mathcal{G}$ are linear and bounded. This problem was solved in [3] via an inertial alternating minimization procedure first proposed in [4] for the case of the strongly coupled problem (1.2).

The above problems and their solution algorithms are limited to two variables which, in addition, must be linearly coupled. These are serious restrictions since models featuring more than two variables and/or nonlinear coupling schemes arise naturally in applications. The purpose of this paper is to address simultaneously these restrictions by proposing a parallel algorithm for solving systems of monotone inclusions involving an arbitrary number of variables and nonlinear coupling. The breadth and flexibility of this framework will be illustrated through applications in the areas of evolution inclusions, best approximation, and network flows.

We now state our problem formulation and our standing assumptions. 
Problem 1.1 Let $\left(\mathcal{H}_{i}\right)_{1 \leq i \leq m}$ be real Hilbert spaces, where $m \geq 2$. For every $i \in\{1, \ldots, m\}$, let $A_{i}: \mathcal{H}_{i} \rightarrow 2^{\mathcal{H}_{i}}$ be maximal monotone and let $B_{i}: \mathcal{H}_{1} \times \cdots \times \mathcal{H}_{m} \rightarrow \mathcal{H}_{i}$. It is assumed that there exists $\beta \in] 0,+\infty[$ such that

$$
\begin{aligned}
& \left(\forall\left(x_{1}, \ldots, x_{m}\right) \in \mathcal{H}_{1} \times \cdots \times \mathcal{H}_{m}\right)\left(\forall\left(y_{1}, \ldots, y_{m}\right) \in \mathcal{H}_{1} \times \cdots \times \mathcal{H}_{m}\right) \\
& \sum_{i=1}^{m}\left\langle B_{i}\left(x_{1}, \ldots, x_{m}\right)-B_{i}\left(y_{1}, \ldots, y_{m}\right) \mid x_{i}-y_{i}\right\rangle \geq \beta \sum_{i=1}^{m}\left\|B_{i}\left(x_{1}, \ldots, x_{m}\right)-B_{i}\left(y_{1}, \ldots, y_{m}\right)\right\|^{2} .
\end{aligned}
$$

The problem is to

$$
\text { find } x_{1} \in \mathcal{H}_{1}, \ldots, x_{m} \in \mathcal{H}_{m} \text { such that }\left\{\begin{array}{c}
0 \in A_{1} x_{1}+B_{1}\left(x_{1}, \ldots, x_{m}\right) \\
\vdots \\
0 \in A_{m} x_{m}+B_{m}\left(x_{1}, \ldots, x_{m}\right),
\end{array}\right.
$$

under the assumption that such points exist.

In abstract terms, the system of inclusions in (1.7) models an equilibrium involving $m$ variables in different Hilbert spaces. The $i$ th inclusion in this system is a perturbation of the basic inclusion $0 \in A_{i} x_{i}$ by addition of the coupling term $B_{i}\left(x_{1}, \ldots, x_{m}\right)$. Our analysis captures various linear and nonlinear coupling schemes. If

$$
(\forall i \in\{1, \ldots, m\}) \quad \mathcal{H}_{i}=\mathcal{H} \quad \text { and } \quad(\forall x \in \mathcal{H}) \quad B_{i}(x, \ldots, x)=0,
$$

then Problem 1.1 is a relaxation of the standard problem [20,33] of finding a common zero of the operators $\left(A_{i}\right)_{1 \leq i \leq m}$, i.e., of solving the inclusion $0 \in \bigcap_{i=1}^{m} A_{i} x$. In particular, if $m=2$, $\mathcal{H}_{1}=\mathcal{H}_{2}=\mathcal{H}, B_{1}=-B_{2}:\left(x_{1}, x_{2}\right) \mapsto x_{1}-x_{2}$, and $\beta=1 / 2$, then Problem 1.1 reverts to (1.1). On the other hand, if $m=2, A_{1}=\partial f_{1}, A_{2}=\partial f_{2}, B_{1}:\left(x_{1}, x_{2}\right) \mapsto L_{1}^{*}\left(L_{1} x_{1}-L_{2} x_{2}\right)$, $B_{2}:\left(x_{1}, x_{2}\right) \mapsto-L_{2}^{*}\left(L_{1} x_{1}-L_{2} x_{2}\right)$, and $\beta=\left(\left\|L_{1}\right\|^{2}+\left\|L_{2}\right\|^{2}\right)^{-1}$, then Problem 1.1 reverts to (1.5). Generally speaking, (1.7) covers coupled problems involving minimizations, variational inequalities, saddle points, or evolution inclusions, depending on the type of the maximal monotone operators $\left(A_{i}\right)_{1 \leq i \leq m}$.

The paper is organized as follows. In Section 2, we present our algorithm for solving Problem 1.1 and prove its convergence. Applications to systems of evolution inclusions are treated in Section 3. Finally, Section 4 is devoted to variational formulations deriving from Problem 1.1 and features applications to best approximation and network flows.

Notation. Throughout, $\mathcal{H}$ and $\left(\mathcal{H}_{i}\right)_{1 \leq i \leq m}$ are real Hilbert spaces. Their scalar products are denoted by $\langle\cdot \mid \cdot\rangle$ and the associated norms by $\|\cdot\|$. The symbols $\rightarrow$ and $\rightarrow$ denote respectively weak and strong convergence, Id denotes the identity operator, and $L^{*}$ denotes the adjoint of a bounded linear operator $L$. The indicator function of a subset $C$ of $\mathcal{H}$ is

$$
\iota_{C}: x \mapsto \begin{cases}0, & \text { if } x \in C ; \\ +\infty, & \text { if } x \notin C,\end{cases}
$$

and the distance from $x \in \mathcal{H}$ to $C$ is $d_{C}(x)=\inf _{y \in C}\|x-y\|$; if $C$ is nonempty closed and convex, the projection of $x$ onto $C$ is the unique point $P_{C} x$ in $C$ such that $\left\|x-P_{C} x\right\|=d_{C}(x)$. We denote by $\Gamma_{0}(\mathcal{H})$ the class of lower semicontinuous convex functions $\left.\left.f: \mathcal{H} \rightarrow\right]-\infty,+\infty\right]$ which are proper 
in the sense that $\operatorname{dom} f=\{x \in \mathcal{H} \mid f(x)<+\infty\} \neq \varnothing$. The subdifferential of $f \in \Gamma_{0}(\mathcal{H})$ is the maximal monotone operator

$$
\partial f: \mathcal{H} \rightarrow 2^{\mathcal{H}}: x \mapsto\{u \in \mathcal{H} \mid(\forall y \in \mathcal{H})\langle y-x \mid u\rangle+f(x) \leq f(y)\} .
$$

We denote by gra $A=\{(x, u) \in \mathcal{H} \times \mathcal{H} \mid u \in A x\}$ the graph of a set-valued operator $A: \mathcal{H} \rightarrow$ $2^{\mathcal{H}}$, by $\operatorname{dom} A=\{x \in \mathcal{H} \mid A x \neq \varnothing\}$ its domain, and by $J_{A}=(\operatorname{Id}+A)^{-1}$ its resolvent. If $A$ is monotone, then $J_{A}$ is single-valued and nonexpansive and, furthermore, if $A$ is maximal monotone, then $\operatorname{dom} J_{A}=\mathcal{H}$. For complements and further background on convex analysis and monotone operator theory, see $[5,15,44,46,48]$.

\section{Algorithm}

Let us start with a characterization of the solutions to Problem 1.1.

Proposition 2.1 Let $\left(x_{i}\right)_{1 \leq i \leq m} \in \mathcal{H}_{1} \times \cdots \times \mathcal{H}_{m}$, let $\left(\lambda_{i}\right)_{1 \leq i \leq m} \in\left[0,1\left[^{m}\right.\right.$, and let $\left.\gamma \in\right] 0,+\infty[$. Then $\left(x_{i}\right)_{1 \leq i \leq m}$ solves Problem 1.1 if and only if

$$
(\forall i \in\{1, \ldots, m\}) \quad x_{i}=\lambda_{i} x_{i}+\left(1-\lambda_{i}\right) J_{\gamma A_{i}}\left(x_{i}-\gamma B_{i}\left(x_{1}, \ldots, x_{m}\right)\right) .
$$

Proof. Let $i \in\{1, \ldots, m\}$. Then, since $B_{i}$ is single-valued,

$$
\begin{aligned}
0 \in A_{i} x_{i}+B_{i}\left(x_{1}, \ldots, x_{m}\right) & \Leftrightarrow x_{i}-\gamma B_{i}\left(x_{1}, \ldots, x_{m}\right) \in x_{i}+\gamma A_{i} x_{i} \\
& \Leftrightarrow x_{i}=J_{\gamma A_{i}}\left(x_{i}-\gamma B_{i}\left(x_{1}, \ldots, x_{m}\right)\right) \\
& \Leftrightarrow x_{i}=x_{i}+\left(1-\lambda_{i}\right)\left(J_{\gamma A_{i}}\left(x_{i}-\gamma B_{i}\left(x_{1}, \ldots, x_{m}\right)\right)-x_{i}\right),
\end{aligned}
$$

and we obtain (2.1).

The above characterization suggests the following algorithm, which constructs $m$ sequences $\left(\left(x_{i, n}\right)_{n \in \mathbb{N}}\right)_{1 \leq i \leq m}$. Recall that $\beta$ is the constant appearing in (1.6).

Algorithm 2.2 Fix $\varepsilon \in] 0, \min \{1, \beta\}\left[,\left(\gamma_{n}\right)_{n \in \mathbb{N}}\right.$ in $[\varepsilon, 2 \beta-\varepsilon],\left(\lambda_{n}\right)_{n \in \mathbb{N}}$ in $[0,1-\varepsilon]$, and $\left(x_{i, 0}\right)_{1 \leq i \leq m}$ $\in \mathcal{H}_{1} \times \cdots \times \mathcal{H}_{m}$. Set, for every $n \in \mathbb{N}$,

$$
\left\{\begin{aligned}
x_{1, n+1} & =\lambda_{1, n} x_{1, n}+\left(1-\lambda_{1, n}\right)\left(J_{\gamma_{n} A_{1, n}}\left(x_{1, n}-\gamma_{n}\left(B_{1, n}\left(x_{1, n}, \ldots, x_{m, n}\right)+b_{1, n}\right)\right)+a_{1, n}\right) \\
& \vdots \\
x_{m, n+1} & =\lambda_{m, n} x_{m, n}+\left(1-\lambda_{m, n}\right)\left(J_{\gamma_{n} A_{m, n}}\left(x_{m, n}-\gamma_{n}\left(B_{m, n}\left(x_{1, n}, \ldots, x_{m, n}\right)+b_{m, n}\right)\right)+a_{m, n}\right),
\end{aligned}\right.
$$

where, for every $i \in\{1, \ldots, m\}$, the following hold.

(i) $\left(A_{i, n}\right)_{n \in \mathbb{N}}$ are maximal monotone operators from $\mathcal{H}_{i}$ to $2^{\mathcal{H}_{i}}$ such that

$$
(\forall \rho \in] 0,+\infty[) \sum_{n \in \mathbb{N}} \sup _{\|y\| \leq \rho}\left\|J_{\gamma_{n} A_{i, n}} y-J_{\gamma_{n} A_{i}} y\right\|<+\infty .
$$

(ii) $\left(B_{i, n}\right)_{n \in \mathbb{N}}$ are operators from $\mathcal{H}_{1} \times \cdots \times \mathcal{H}_{m}$ to $\mathcal{H}_{i}$ such that 
(a) the operators $\left(B_{i, n}-B_{i}\right)_{n \in \mathbb{N}}$ are Lipschitz-continuous with respective constants $\left(\kappa_{i, n}\right)_{n \in \mathbb{N}}$ in $] 0,+\infty\left[\right.$ satisfying $\sum_{n \in \mathbb{N}} \kappa_{i, n}<+\infty$; and

(b) there exists $\boldsymbol{z} \in \mathcal{H}_{1} \times \cdots \times \mathcal{H}_{m}$, independent of $i$, such that $(\forall n \in \mathbb{N}) B_{i, n} \boldsymbol{z}=B_{i} \boldsymbol{z}$.

(iii) $\left(a_{i, n}\right)_{n \in \mathbb{N}}$ and $\left(b_{i, n}\right)_{n \in \mathbb{N}}$ are sequences in $\mathcal{H}_{i}$ such that $\sum_{n \in \mathbb{N}}\left\|a_{i, n}\right\|<+\infty$ and $\sum_{n \in \mathbb{N}}\left\|b_{i, n}\right\|<$ $+\infty$.

(iv) $\left(\lambda_{i, n}\right)_{n \in \mathbb{N}}$ is a sequence in $\left[0,1\left[\right.\right.$ such that $\sum_{n \in \mathbb{N}}\left|\lambda_{i, n}-\lambda_{n}\right|<+\infty$.

Conditions (i) and (ii) describe the types of approximations to the original operators $\left(A_{i}\right)_{1 \leq i \leq m}$ and $\left(B_{i}\right)_{1 \leq i \leq m}$ which can be utilized. Condition (iii) quantifies the tolerance which is allowed in the implementation of these approximations (see [25, 31, 32] for specific examples), while (iv) quantifies that allowed in the agent-dependent departure from the global relaxation scheme. The parallel nature of Algorithm 2.2 stems from the fact that the $m$ evaluations of the resolvent operators in (2.3) can be performed independently and, therefore, simultaneously on concurrent processors.

Our asymptotic analysis of Algorithm 2.2 will be based on Theorem 2.8 below on the convergence of the forward-backward algorithm. First, we need to introduce the notion of demiregularity. This notion captures various properties typically used to establish the strong convergence of dynamical systems, e.g., compactness [18], bounded compactness [8, 21, 22], uniform monotonicity [22, 24, 48], uniform convexity [26, 29, 34, 46], compactness of resolvents [30], and demicompactness [38, 47]. In the case of at most single-valued operators, demiregularity captures standard regularity properties used in nonlinear analysis [48, Definition 27.1].

Definition 2.3 An operator $A: \mathcal{H} \rightarrow 2^{\mathcal{H}}$ is demiregular at $y \in \operatorname{dom} A$ if, for every sequence $\left(\left(y_{n}, v_{n}\right)\right)_{n \in \mathbb{N}}$ in gra $A$ and every $v \in A y$, we have

$$
\left\{\begin{array}{l}
y_{n} \rightarrow y \\
v_{n} \rightarrow v
\end{array} \quad \Rightarrow \quad y_{n} \rightarrow y .\right.
$$

Proposition 2.4 Let $A: \mathcal{H} \rightarrow 2^{\mathcal{H}}$, let $y \in \operatorname{dom} A$, and let $\mathcal{C}$ be the set of all nondecreasing functions from $[0,+\infty[$ to $[0,+\infty]$ that vanish only at 0 . Suppose that one of the following holds.

(i) $A$ is uniformly monotone at $y$, i.e., there exists $\phi \in \mathcal{C}$ such that

$$
(\forall v \in A y)(\forall(x, u) \in \operatorname{gra} A) \quad\langle x-y \mid u-v\rangle \geq \phi(\|x-y\|) .
$$

(ii) $A$ is uniformly monotone, i.e., there exists $\phi \in \mathcal{C}$ such that (2.6) holds for every $y \in \operatorname{dom} A$.

(iii) $A$ is strongly monotone, i.e., there exists $\rho \in] 0,+\infty[$ such that $A-\rho \operatorname{Id}$ is monotone.

(iv) $A=\partial f$, where $f \in \Gamma_{0}(\mathcal{H})$ is uniformly convex at $y[46$, Section 3.4], i.e., there exists $\phi \in \mathcal{C}$ such that

$$
\begin{aligned}
& (\forall \alpha \in] 0,1[)(\forall x \in \operatorname{dom} f) \\
& \qquad f(\alpha x+(1-\alpha) y)+\alpha(1-\alpha) \phi(\|x-y\|) \leq \alpha f(x)+(1-\alpha) f(y) .
\end{aligned}
$$

(v) $A=\partial f$, where $f \in \Gamma_{0}(\mathcal{H})$ is uniformly convex, i.e., there exists $\phi \in \mathcal{C}$ such that (2.7) holds for every $y \in \operatorname{dom} f$. 
(vi) $A=\partial f$, where $f \in \Gamma_{0}(\mathcal{H})$ is strongly convex, i.e., there exists $\left.\rho \in\right] 0,+\infty\left[\right.$ such that $f-\rho\|\cdot\|^{2} / 2$ is convex.

(vii) $A=\partial f$, where $f \in \Gamma_{0}(\mathcal{H})$ and the lower level sets of $f$ are boundedly compact.

(viii) $J_{A}$ is compact, i.e., for every bounded set $C \subset \mathcal{H}$, the closure of $J_{A}(C)$ is compact.

(ix) $\operatorname{dom} A$ is boundedly relatively compact, i.e., the intersection of its closure with every closed ball is compact.

(x) $\mathcal{H}$ is finite-dimensional.

(xi) $A: \mathcal{H} \rightarrow \mathcal{H}$ is single-valued with a single-valued continuous inverse.

(xii) $A$ is single-valued on $\operatorname{dom} A$ and $\operatorname{Id}-A$ demicompact [38], [47, Section 10.4], i.e., for every bounded sequence $\left(x_{n}\right)_{n \in \mathbb{N}}$ in $\operatorname{dom} A$ such that $\left(A x_{n}\right)_{n \in \mathbb{N}}$ converges strongly, $\left(x_{n}\right)_{n \in \mathbb{N}}$ admits a strong cluster point.

Then $A$ is demiregular at $y$.

Proof. Let $\left(\left(y_{n}, v_{n}\right)\right)_{n \in \mathbb{N}}$ be a sequence in gra $A$ and let $v \in A y$ be such that $y_{n} \rightarrow y$ and $v_{n} \rightarrow v$. We must show that $y_{n} \rightarrow y$.

(i): By (2.6), there exists $\phi \in \mathcal{C}$ such that $(\forall n \in \mathbb{N})\left\langle y_{n}-y \mid v_{n}-v\right\rangle \geq \phi\left(\left\|y_{n}-y\right\|\right)$. However, since $y_{n} \rightarrow y$ and $v_{n} \rightarrow v$, we have $\left\langle y_{n}-y \mid v_{n}-v\right\rangle \rightarrow 0$. Therefore, appealing to the properties of $\phi$, we conclude that $\left\|y_{n}-y\right\| \rightarrow 0$.

(ii) $\Rightarrow(\mathrm{i})$ : Clear.

(iii) $\Rightarrow$ (ii): Indeed, $A$ is uniformly monotone with $\phi: t \mapsto \rho t^{2}$.

(iv) $\Rightarrow($ i): See $[46$, Section 3.4].

$(\mathrm{v}) \Rightarrow(\mathrm{iv})$ : Clear.

$(\mathrm{vi}) \Rightarrow(\mathrm{v})$ : Indeed, $f$ is uniformly convex with $\phi: t \mapsto \rho t^{2} / 2$.

(vii): Since $\left\langle y_{n}-y \mid v_{n}\right\rangle \rightarrow 0$, there exists $\left.\rho \in\right] 0,+\infty\left[\operatorname{such}_{\text {that }} \sup _{n \in \mathbb{N}}\left\langle y_{n}-y \mid v_{n}\right\rangle \leq \rho\right.$. Hence, since $y \in \operatorname{dom} \partial f \subset \operatorname{dom} f,(1.10)$ yields $(\forall n \in \mathbb{N}) f\left(y_{n}\right) \leq f(y)+\left\langle y_{n}-y \mid v_{n}\right\rangle \leq f(y)+\rho<+\infty$. Altogether, $\left(y_{n}\right)_{n \in \mathbb{N}}$ is bounded and lies in a lower level set of $f$. It therefore lies in a compact set. However, since weak convergence and strong convergence coincide for sequences in compact sets, we conclude that $y_{n} \rightarrow y$.

(viii): We have $(\forall n \in \mathbb{N})\left(y_{n}, v_{n}\right) \in \operatorname{gra} A \Rightarrow\left(v_{n}+y_{n}\right)-y_{n} \in A y_{n} \Rightarrow y_{n}=J_{A}\left(v_{n}+y_{n}\right)$. Since $\left(v_{n}+y_{n}\right)_{n \in \mathbb{N}}$ converge weakly, it lies in a bounded set $C$. Thus, $\left(y_{n}\right)_{n \in \mathbb{N}}$ lies in $J_{A}(C)$, which has compact closure. Hence $y_{n} \rightarrow y \Rightarrow y_{n} \rightarrow y$.

(ix) $\Rightarrow$ (viii): Let $C \subset \mathcal{H}$ be bounded. Then $J_{A}(C) \subset J_{A}(\mathcal{H})=\operatorname{dom} A$ and, by nonexpansivity of $J_{A}$ [5, Proposition 3.5.3], $J_{A}(C)$ is bounded. Altogether, $J_{A}(C)$ has compact closure.

$(\mathrm{x}) \Rightarrow(\mathrm{ix})$ : Clear.

(xi): Since $A y_{n}=v_{n} \rightarrow v=A y$, we have $y_{n}=A^{-1} v_{n} \rightarrow A^{-1} v=y$. 
(xii): Since $\left(y_{n}\right)_{n \in \mathbb{N}}$ converges weakly, it is bounded. In addition, $\left(A y_{n}\right)_{n \in \mathbb{N}}=\left(v_{n}\right)_{n \in \mathbb{N}}$ converges strongly. Hence, by demicompactness of $\mathrm{Id}-A,\left(y_{n}\right)_{n \in \mathbb{N}}$ has a strong cluster point $x$ and, since $y_{n} \rightarrow y$, we must have $x=y$. Now suppose that $y_{n} \not \supset y$. Then, there exist $\left.\varepsilon \in\right] 0,+\infty[$ and a subsequence $\left(y_{k_{n}}\right)_{n \in \mathbb{N}}$ such that

$$
(\forall n \in \mathbb{N}) \quad\left\|y_{k_{n}}-y\right\| \geq \varepsilon .
$$

However, since $y_{k_{n}} \rightarrow y$ and $\left(A y_{k_{n}}\right)_{n \in \mathbb{N}}$ converges strongly, arguing as above, we can extract a further subsequence $\left(y_{l_{k_{n}}}\right)_{n \in \mathbb{N}}$ such that $y_{l_{k_{n}}} \rightarrow y$, which contradicts (2.8). Therefore, $y_{n} \rightarrow y$. $\square$

Next, we recall the notion of cocoercivity.

Definition 2.5 Let $\chi \in] 0,+\infty[$. An operator $B: \mathcal{H} \rightarrow \mathcal{H}$ is $\chi$-cocoercive if $\chi B$ is firmly nonexpansive, i.e.,

$$
(\forall x \in \mathcal{H})(\forall y \in \mathcal{H}) \quad\langle x-y \mid B x-B y\rangle \geq \chi\|B x-B y\|^{2} .
$$

Firmly nonexpansive operators include resolvents of maximal monotone operators, proximity operators, and projectors onto nonempty closed convex sets. In addition, the Yosida approximation of a maximal monotone operator of index $\chi$ is $\chi$-cocoercive [2] (further examples of cocoercive operators can be found in [49]). It is clear from (2.9) that, if $B$ is $\chi$-cocoercive, then it is $\chi^{-1}$ Lipschitz continuous. The next lemma, which provides a converse implication, supplies us with another important instance of cocoercive operator (see also [27]).

Lemma 2.6 [7, Corollaire 10] Let $\varphi: \mathcal{H} \rightarrow \mathbb{R}$ be a differentiable convex function and let $\tau \in] 0,+\infty[$. Suppose that $\nabla \varphi$ is $\tau$-Lipschitz continuous. Then $\nabla \varphi$ is $\tau^{-1}$-cocoercive.

We shall also use the following fact.

Lemma 2.7 [22, Lemma 2.3] Let $\chi \in] 0,+\infty[$, let $B: \mathcal{H} \rightarrow \mathcal{H}$ be a $\chi$-cocoercive operator, and let $\gamma \in] 0,2 \chi[$. Then $\mathrm{Id}-\gamma B$ is nonexpansive.

We are now ready to record some convergence properties of the forward-backward algorithm, which are of interest in their own right. The forward-backward algorithm finds its roots in the projected gradient method [34] and certain methods for solving variational inequalities [6, 16, 35, 43] (see also the bibliography of [22] for more recent developments).

Theorem 2.8 Let $(\mathcal{H}, \||\cdot|||)$ be a real Hilbert space, let $\chi \in] 0,+\infty\left[\right.$, let $\boldsymbol{A}: \mathcal{H} \rightarrow 2^{\mathcal{H}}$ be a maximal monotone operator, and let $\boldsymbol{B}: \mathcal{H} \rightarrow \mathcal{H}$ be a $\chi$-cocoercive operator such that

$$
\boldsymbol{Z}=(\boldsymbol{A}+\boldsymbol{B})^{-1}(\mathbf{0}) \neq \varnothing .
$$

Fix $\varepsilon \in] 0, \min \{1, \chi\}\left[\right.$, let $\left(\gamma_{n}\right)_{n \in \mathbb{N}}$ be a sequence in $[\varepsilon, 2 \chi-\varepsilon]$, let $\left(\lambda_{n}\right)_{n \in \mathbb{N}}$ be a sequence in $[0,1-\varepsilon]$, and let $\left(\boldsymbol{a}_{n}\right)_{n \in \mathbb{N}}$ and $\left(\boldsymbol{b}_{n}\right)_{n \in \mathbb{N}}$ be sequences in $\mathcal{H}$ such that $\sum_{n \in \mathbb{N}}\left|\left\|\boldsymbol{a}_{n} \mid\right\|<+\infty\right.$ and $\left.\sum_{n \in \mathbb{N}}\right|\left\|\boldsymbol{b}_{n} \mid\right\|<$ $+\infty$. Fix $\boldsymbol{x}_{0} \in \mathcal{H}$ and, for every $n \in \mathbb{N}$, set

$$
\boldsymbol{x}_{n+1}=\lambda_{n} \boldsymbol{x}_{n}+\left(1-\lambda_{n}\right)\left(J_{\gamma_{n} \boldsymbol{A}}\left(\boldsymbol{x}_{n}-\gamma_{n}\left(\boldsymbol{B} x_{n}+\boldsymbol{b}_{n}\right)\right)+\boldsymbol{a}_{n}\right) \text {. }
$$

Then the following hold for some $\boldsymbol{x} \in \boldsymbol{Z}$.

(i) $\boldsymbol{x}_{n} \rightarrow \boldsymbol{x}$. 
(ii) $\boldsymbol{B} \boldsymbol{x}_{n} \rightarrow \boldsymbol{B} \boldsymbol{x}$.

(iii) $\boldsymbol{x}_{n}-J_{\gamma_{n} \boldsymbol{A}}\left(\boldsymbol{x}_{n}-\gamma_{n} \boldsymbol{B} \boldsymbol{x}_{n}\right) \rightarrow \mathbf{0}$.

(iv) Suppose that one of the following is satisfied.

(a) $\boldsymbol{A}$ is demiregular at $\boldsymbol{x}$ (see Proposition 2.4 for special cases).

(b) $\boldsymbol{B}$ is demiregular at $\boldsymbol{x}$ (see Proposition 2.4 for special cases).

(c) int $\boldsymbol{Z} \neq \varnothing$.

Then $\boldsymbol{x}_{n} \rightarrow \boldsymbol{x}$.

Proof. For every $n \in \mathbb{N}$, set

$$
\begin{aligned}
& \boldsymbol{T}_{1, n}=J_{\gamma_{n} \boldsymbol{A}}, \boldsymbol{T}_{2, n}=\mathbf{I d}-\gamma_{n} \boldsymbol{B}, \\
& \qquad \boldsymbol{e}_{1, n}=\boldsymbol{a}_{n}, \boldsymbol{e}_{2, n}=-\gamma_{n} \boldsymbol{b}_{n}, \mu_{n}=1-\lambda_{n}, \beta_{1, n}=2, \text { and } \beta_{2, n}=\frac{2 \chi}{\gamma_{n}} .
\end{aligned}
$$

Then $\sum_{n \in \mathbb{N}} \mu_{n}||\left|\boldsymbol{e}_{1, n}\right|\left\|<+\infty, \sum_{n \in \mathbb{N}} \mu_{n}||\left|\boldsymbol{e}_{2, n}\right|\right\|<+\infty$, and, by [22, Equation (6.5)], $\boldsymbol{Z}=$ $\bigcap_{n \in \mathbb{N}} \operatorname{Fix} \boldsymbol{T}_{1, n} \boldsymbol{T}_{2, n}$. Moreover, as seen in [22, Section 6], $\left(1-\beta_{1, n}\right) \mathbf{I} \mathbf{d}+\beta_{1, n} \boldsymbol{T}_{1, n}$ and $\left(1-\beta_{2, n}\right) \mathbf{I d}+$ $\beta_{2, n} \boldsymbol{T}_{2, n}$ are nonexpansive, and (2.11) can be rewritten as

$$
\boldsymbol{x}_{n+1}=\boldsymbol{x}_{n}+\mu_{n}\left(\boldsymbol{T}_{1, n}\left(\boldsymbol{T}_{2, n} x_{n}+\boldsymbol{e}_{2, n}\right)+\boldsymbol{e}_{1, n}-\boldsymbol{x}_{n}\right),
$$

which is precisely the iteration governing [22, Algorithm 1.2], where $m=2$.

(i): [22, Corollary 6.5].

(ii)\&(iii): We derive from (2.13), [22, Remark 3.4], and our assumptions on $\left(\lambda_{n}\right)_{n \in \mathbb{N}}$ and $\left(\gamma_{n}\right)_{n \in \mathbb{N}}$ that $\left(\mathbf{I d}-\boldsymbol{T}_{2, n}\right) \boldsymbol{x}_{n}-\left(\mathbf{I d}-\boldsymbol{T}_{2, n}\right) \boldsymbol{x} \rightarrow \mathbf{0}$ and, in turn, that $\boldsymbol{B} \boldsymbol{x}_{n} \rightarrow \boldsymbol{B} \boldsymbol{x}$. Likewise, [22, Remark 3.4] yields $\boldsymbol{x}_{n}-\boldsymbol{T}_{1, n} \boldsymbol{T}_{2, n} \boldsymbol{x}_{n} \rightarrow \mathbf{0}$ and, therefore, $\boldsymbol{x}_{n}-J_{\gamma_{n} \boldsymbol{A}}\left(\boldsymbol{x}_{n}-\gamma_{n} \boldsymbol{B} \boldsymbol{x}_{n}\right) \rightarrow \mathbf{0}$.

(iv)(a): Set $\boldsymbol{v}=-\boldsymbol{B} \boldsymbol{x}$ and

$$
(\forall n \in \mathbb{N}) \quad\left\{\begin{array}{l}
\boldsymbol{y}_{n}=J_{\gamma_{n}} \boldsymbol{A}\left(\boldsymbol{x}_{n}-\gamma_{n} \boldsymbol{B} \boldsymbol{x}_{n}\right) \\
\boldsymbol{v}_{n}=\gamma_{n}^{-1}\left(\boldsymbol{x}_{n}-\boldsymbol{y}_{n}\right)-\boldsymbol{B} \boldsymbol{x}_{n} .
\end{array}\right.
$$

On the one hand, we have $(\forall n \in \mathbb{N})\left(\boldsymbol{y}_{n}, \boldsymbol{v}_{n}\right) \in$ gra $\boldsymbol{A}$. On the other hand, we derive from (i) and (iii) that $\boldsymbol{y}_{n} \rightarrow \boldsymbol{x}$. Furthermore, since

$$
(\forall n \in \mathbb{N}) \quad\left\|\boldsymbol{v}_{n}-\boldsymbol{v}\right\| \mid \leq \frac{\left\|\boldsymbol{x}_{n}-\boldsymbol{y}_{n}\right\| \mid}{\gamma_{n}}+\left\|\boldsymbol{B} \boldsymbol{x}_{n}-\boldsymbol{B} \boldsymbol{x}\right\| \|,
$$

it follows from (ii), (iii), and the condition $\inf _{n \in \mathbb{N}} \gamma_{n}>0$ that $\boldsymbol{v}_{n} \rightarrow \boldsymbol{v}$. It then results from Definition 2.3 that $\boldsymbol{y}_{n} \rightarrow \boldsymbol{x}$ and, in turn, from (iii) that $\boldsymbol{x}_{n} \rightarrow \boldsymbol{x}$.

(iv)(b): Set $\boldsymbol{v}=\boldsymbol{B} \boldsymbol{x}$ and $(\forall n \in \mathbb{N}) \boldsymbol{v}_{n}=\boldsymbol{B} \boldsymbol{x}_{n}$. Then (i) yields $\boldsymbol{x}_{n} \rightarrow \boldsymbol{x}$ and (ii) yields $\boldsymbol{v}_{n} \rightarrow \boldsymbol{v}$. It thus follows from Definition 2.3 that $\boldsymbol{x}_{n} \rightarrow \boldsymbol{x}$.

(iv)(c): This follows from (i) and [22, Theorem 3.3(i) \& Lemma 2.8(iv)].

The main results of this section are the following theorems. Let us start with weak convergence. 
Theorem 2.9 Let $\left(\left(x_{i, n}\right)_{n \in \mathbb{N}}\right)_{1 \leq i \leq m}$ be sequences generated by Algorithm 2.2. Then, for every $i \in\{1, \ldots, m\},\left(x_{i, n}\right)_{n \in \mathbb{N}}$ converges weakly to a point $x_{i} \in \mathcal{H}_{i}$, and $\left(x_{i}\right)_{1 \leq i \leq m}$ is a solution to Problem 1.1.

Proof. Throughout the proof, a generic element $\boldsymbol{x}$ in the Cartesian product $\mathcal{H}_{1} \times \cdots \times \mathcal{H}_{m}$ will be expressed in terms of its components as $\boldsymbol{x}=\left(x_{i}\right)_{1 \leq i \leq m}$. We shall show that our algorithmic setting reduces to the situation described in Theorem 2.8(i) in the Hilbert direct sum $\mathcal{H}=\mathcal{H}_{1} \oplus \cdots \oplus \mathcal{H}_{m}$ obtained by endowing $\mathcal{H}_{1} \times \cdots \times \mathcal{H}_{m}$ with the scalar product

$$
\langle\langle\cdot \mid \cdot\rangle\rangle:(\boldsymbol{x}, \boldsymbol{y}) \mapsto \sum_{i=1}^{m}\left\langle x_{i} \mid y_{i}\right\rangle,
$$

with associated norm

$$
\left\|\left|\|\mid\|: \sqrt{\sum_{i=1}^{m}\left\|x_{i}\right\|^{2}} .\right.\right.
$$

To this end, we shall show that the iterations (2.3) can be cast in the form of (2.11). First, define

$$
\boldsymbol{A}: \mathcal{H} \rightarrow 2^{\mathcal{H}}: \boldsymbol{x} \mapsto \underset{i=1}{\times} A_{i} x_{i} \quad \text { and } \quad(\forall n \in \mathbb{N}) \quad \boldsymbol{A}_{n}: \mathcal{H} \rightarrow 2^{\mathcal{H}}: \boldsymbol{x} \mapsto \underset{i=1}{\times} A_{i, n} x_{i}
$$

It follows from the maximal monotonicity of the operators $\left(A_{i}\right)_{1 \leq i \leq m}$, condition (i) in Algorithm 2.2, (2.16), and (2.18) that

$$
\boldsymbol{A} \text { and }\left(\boldsymbol{A}_{n}\right)_{n \in \mathbb{N}} \text { are maximal monotone, }
$$

with resolvents

$$
J_{\boldsymbol{A}}: \mathcal{H} \rightarrow \mathcal{H}: \boldsymbol{x} \mapsto\left(J_{A_{i}} x_{i}\right)_{1 \leq i \leq m} \quad \text { and } \quad(\forall n \in \mathbb{N}) \quad J_{\boldsymbol{A}_{n}}: \mathcal{H} \rightarrow \mathcal{H}: \boldsymbol{x} \mapsto\left(J_{A_{i, n}} x_{i}\right)_{1 \leq i \leq m},
$$

respectively. Moreover, for every $\rho \in] 0,+\infty[$, we derive from (2.17), (2.20), and condition (i) in Algorithm 2.2 that

$$
\begin{aligned}
\sum_{n \in \mathbb{N}} \sup _{\|\boldsymbol{y}\| \leq \rho}\left\|J_{\gamma_{n} \boldsymbol{A}_{n}} \boldsymbol{y}-J_{\gamma_{n} \boldsymbol{A}} \boldsymbol{y}\right\| \| & =\sum_{n \in \mathbb{N}} \sup _{\|\boldsymbol{y}\| \leq \rho} \sqrt{\sum_{i=1}^{m}\left\|J_{\gamma_{n} A_{i, n}} y_{i}-J_{\gamma_{n} A_{i}} y_{i}\right\|^{2}} \\
& \leq \sum_{n \in \mathbb{N}} \sup _{\|\boldsymbol{y}\| \leq \rho} \sum_{i=1}^{m}\left\|J_{\gamma_{n} A_{i, n}} y_{i}-J_{\gamma_{n} A_{i}} y_{i}\right\| \\
& \leq \sum_{i=1}^{m} \sum_{n \in \mathbb{N}} \sup _{\left\|y_{i}\right\| \leq \rho}\left\|J_{\gamma_{n} A_{i, n}} y_{i}-J_{\gamma_{n} A_{i}} y_{i}\right\| \\
& <+\infty
\end{aligned}
$$

Now define

$$
\boldsymbol{B}: \mathcal{H} \rightarrow \mathcal{H}: \boldsymbol{x} \mapsto\left(B_{i} \boldsymbol{x}\right)_{1 \leq i \leq m} \quad \text { and } \quad(\forall n \in \mathbb{N}) \quad \boldsymbol{B}_{n}: \mathcal{H} \rightarrow \mathcal{H}: \boldsymbol{x} \mapsto\left(B_{i, n} \boldsymbol{x}\right)_{1 \leq i \leq m} .
$$

Then (1.7) is equivalent to

$$
\text { find } \boldsymbol{x} \in \boldsymbol{Z}=(\boldsymbol{A}+\boldsymbol{B})^{-1}(\mathbf{0}) \text {. }
$$

Moreover, in the light of (2.16), (2.17), and (2.22), (1.6) becomes

$$
(\forall \boldsymbol{x} \in \mathcal{H})(\forall \boldsymbol{y} \in \mathcal{H}) \quad\langle\langle\boldsymbol{x}-\boldsymbol{y} \mid \boldsymbol{B} \boldsymbol{x}-\boldsymbol{B} \boldsymbol{y}\rangle\rangle \geq \beta\|\| \boldsymbol{B} \boldsymbol{x}-\boldsymbol{B} \boldsymbol{y}\|\|^{2} .
$$


In other words, $\boldsymbol{B}$ is $\beta$-cocoercive. Next, let $n \in \mathbb{N}$ and set

$$
\boldsymbol{c}_{n}=\left(a_{i, n}\right)_{1 \leq i \leq m} \quad \text { and } \quad \boldsymbol{d}_{n}=\left(b_{i, n}\right)_{1 \leq i \leq m} .
$$

We deduce from (2.17) and condition (iii) in Algorithm 2.2 that

$$
\sum_{k \in \mathbb{N}}\|\| \boldsymbol{c}_{k}\left\|\mid \leq \sum_{k \in \mathbb{N}} \sqrt{\sum_{i=1}^{m}\left\|a_{i, k}\right\|^{2}} \leq \sum_{i=1}^{m} \sum_{k \in \mathbb{N}}\right\| a_{i, k} \|<+\infty
$$

and, likewise, that

$$
\sum_{k \in \mathbb{N}}\left|\left\|\boldsymbol{d}_{k} \mid\right\|<+\infty\right.
$$

Now set

$$
\boldsymbol{x}_{n}=\left(x_{i, n}\right)_{1 \leq i \leq m} \quad \text { and } \quad \boldsymbol{\Lambda}_{n}: \mathcal{H} \rightarrow \mathcal{H}: \boldsymbol{x} \mapsto\left(\lambda_{i, n} x_{i}\right)_{1 \leq i \leq m} .
$$

It follows from (2.17) and condition (iv) in Algorithm 2.2 that

$$
\left\|\left|\boldsymbol{\Lambda}_{n}\right|\right\|=\max _{1 \leq i \leq m} \lambda_{i, n} \leq 1 \quad \text { and } \quad\left\|\mathbf{I d}-\boldsymbol{\Lambda}_{n}\right\| \mid=1-\min _{1 \leq i \leq m} \lambda_{i, n} \leq 1 .
$$

Hence,

$$
||\left|\boldsymbol{\Lambda}_{n}\right|||+|| \mathbf{I d}-\boldsymbol{\Lambda}_{n} \mid \|=1+\max _{1 \leq i \leq m}\left(\lambda_{i, n}-\lambda_{n}\right)-\min _{1 \leq i \leq m}\left(\lambda_{i, n}-\lambda_{n}\right) \leq 1+\tau_{n},
$$

where

$$
\tau_{n}=2 \max _{1 \leq i \leq m}\left|\lambda_{i, n}-\lambda_{n}\right| .
$$

We observe that, by virtue of condition (iv) in Algorithm 2.2,

$$
\sum_{k \in \mathbb{N}} \tau_{k}=2 \sum_{k \in \mathbb{N}} \max _{1 \leq i \leq m}\left|\lambda_{i, k}-\lambda_{k}\right| \leq 2 \sum_{i=1}^{m} \sum_{k \in \mathbb{N}}\left|\lambda_{i, k}-\lambda_{k}\right|<+\infty .
$$

Moreover, in view of (2.20), (2.22), (2.25), and (2.28), the iterations (2.3) are equivalent to

$$
\boldsymbol{x}_{n+1}=\boldsymbol{\Lambda}_{n} \boldsymbol{x}_{n}+\left(\mathbf{I d}-\boldsymbol{\Lambda}_{n}\right)\left(J_{\gamma_{n} \boldsymbol{A}_{n}}\left(\boldsymbol{x}_{n}-\gamma_{n}\left(\boldsymbol{B}_{n} \boldsymbol{x}_{n}+\boldsymbol{d}_{n}\right)\right)+\boldsymbol{c}_{n}\right) .
$$

Now define

$$
\boldsymbol{D}_{n}=\boldsymbol{B}_{n}-\boldsymbol{B} .
$$

It follows from condition (ii)(a) in Algorithm 2.2, (2.17), and (2.22) that $\boldsymbol{D}_{n}$ is Lipschitz continuous with constant $\kappa_{n}=\sqrt{\sum_{i=1}^{m} \kappa_{i, n}^{2}}$ and that

$$
\sum_{k \in \mathbb{N}} \kappa_{k}=\sum_{k \in \mathbb{N}} \sqrt{\sum_{i=1}^{m} \kappa_{i, k}^{2}} \leq \sum_{i=1}^{m} \sum_{k \in \mathbb{N}} \kappa_{i, k}<+\infty
$$

Furthermore, set

$$
\boldsymbol{b}_{n}=\boldsymbol{D}_{n} \boldsymbol{x}_{n}+\boldsymbol{d}_{n}
$$

and let $\boldsymbol{x} \in \boldsymbol{Z}$. Then

$$
\begin{aligned}
& ||\left|\boldsymbol{b}_{n}\right||| \leq|| \boldsymbol{D}_{n} \boldsymbol{x}_{n}|||+||| \boldsymbol{d}_{n}|| \mid \\
& \leq||\left|\boldsymbol{D}_{n} \boldsymbol{x}_{n}-\boldsymbol{D}_{n} \boldsymbol{x}\right|||+|| \boldsymbol{D}_{n} \boldsymbol{x}-\boldsymbol{D}_{n} \boldsymbol{z}|||+||| \boldsymbol{d}_{n}|| \mid \\
& \leq \kappa_{n}\left(\left|\left\|\boldsymbol{x}_{n}-\boldsymbol{x}\right\|\right|+|\| \boldsymbol{x}-\boldsymbol{z}|||\right)+\|\left|\boldsymbol{d}_{n}\right|||,
\end{aligned}
$$


where $\boldsymbol{z}$ is provided by assumption (ii)(b) in Algorithm 2.2. We now set

$$
\boldsymbol{T}_{n}=\mathbf{I d}-\gamma_{n} \boldsymbol{B} \text { and } \boldsymbol{e}_{n}=J_{\gamma_{n} \boldsymbol{A}_{n}}\left(\boldsymbol{T}_{n} \boldsymbol{x}\right)-\boldsymbol{x} .
$$

On the one hand, the inequality $\sup _{k \in \mathbb{N}} \gamma_{k} \leq 2 \beta$ yields

$$
\left\|\boldsymbol{T}_{n} \boldsymbol{x} \mid\right\| \leq \rho, \quad \text { where } \quad \rho=\|\| \boldsymbol{x}\||+2 \beta|\| \boldsymbol{B} \boldsymbol{x} \mid \| .
$$

On the other hand, since $\boldsymbol{x}$ is a solution to Problem 1.1, Proposition 2.1, (2.20), and (2.22) supply

$$
\boldsymbol{x}=J_{\gamma_{n} \boldsymbol{A}}\left(\boldsymbol{T}_{n} \boldsymbol{x}\right) .
$$

Therefore, (2.38), (2.39), and (2.21) imply that

$$
\sum_{k \in \mathbb{N}}\left|\left\|\boldsymbol { e } _ { k } \left|\left\|\left|=\sum_{k \in \mathbb{N}}\left\|\left|J_{\gamma_{k}} \boldsymbol{A}_{k}\left(\boldsymbol{T}_{k} \boldsymbol{x}\right)-\boldsymbol{x}\left\|\left|=\sum_{k \in \mathbb{N}}\right|\right\| J_{\gamma_{k}} \boldsymbol{A}_{k}\left(\boldsymbol{T}_{k} \boldsymbol{x}\right)-J_{\gamma_{k}} \boldsymbol{A}\left(\boldsymbol{T}_{k} \boldsymbol{x}\right)\right|\right\|<+\infty\right.\right.\right.\right.\right.
$$

In addition, (2.34), (2.36), and (2.38) yield

$$
J_{\gamma_{n} \boldsymbol{A}_{n}}\left(\boldsymbol{x}_{n}-\gamma_{n}\left(\boldsymbol{B}_{n} \boldsymbol{x}_{n}+\boldsymbol{d}_{n}\right)\right)-\boldsymbol{x}=J_{\gamma_{n} \boldsymbol{A}_{n}}\left(\boldsymbol{T}_{n} \boldsymbol{x}_{n}-\gamma_{n} \boldsymbol{b}_{n}\right)-J_{\gamma_{n} \boldsymbol{A}_{n}}\left(\boldsymbol{T}_{n} \boldsymbol{x}\right)+\boldsymbol{e}_{n} .
$$

Since $J_{\gamma_{n} \boldsymbol{A}}$ is nonexpansive as a resolvent (see [5, Proposition 3.5.3] or [15, Proposition 2.2.iii)]) and $\boldsymbol{T}_{n}$ is nonexpansive by Lemma 2.7, we derive from (2.42) and (2.37) that

$$
\begin{aligned}
& ||\left|J_{\gamma_{n} \boldsymbol{A}_{n}}\left(\boldsymbol{x}_{n}-\gamma_{n}\left(\boldsymbol{B}_{n} \boldsymbol{x}_{n}+\boldsymbol{d}_{n}\right)\right)-\boldsymbol{x}\right|\left\||\leq||| J_{\gamma_{n}} \boldsymbol{A}_{n}\left(\boldsymbol{T}_{n} \boldsymbol{x}_{n}-\gamma_{n} \boldsymbol{b}_{n}\right)-J_{\gamma_{n} \boldsymbol{A}_{n}}\left(\boldsymbol{T}_{n} \boldsymbol{x}\right)\left|\left\|+||\left|\boldsymbol{e}_{n}\right|\right\|\right|\right. \\
& \leq\left\||| \boldsymbol{T}_{n} \boldsymbol{x}_{n}-\gamma_{n} \boldsymbol{b}_{n}-\boldsymbol{T}_{n} \boldsymbol{x}\right\|\left|+\left\|\boldsymbol{e}_{n}\right\|\right| \\
& \leq||\left|\boldsymbol{x}_{n}-\boldsymbol{x}\right| \|+\gamma_{n}||\left|\boldsymbol{b}_{n}\right|||+|| \boldsymbol{e}_{n}|| \mid \\
& \leq||\left|\boldsymbol{x}_{n}-\boldsymbol{x}\right|||+2 \beta||\left|\boldsymbol{b}_{n}\right|||+\left|\| \boldsymbol{e}_{n}\right||| \\
& \leq\left(1+2 \beta \kappa_{n}\right)||\left|\boldsymbol{x}_{n}-\boldsymbol{x}\right|||+2 \beta \kappa_{n}|\| \boldsymbol{x}-\boldsymbol{z}||| \\
& +2 \beta\left\|\left|\boldsymbol { d } _ { n } \left\|\left|+\left\|\boldsymbol{e}_{n}\right\|\right|\right.\right.\right. \text {. }
\end{aligned}
$$

Thus, it results from (2.33), (2.43), (2.30), and (2.29) that

$$
\begin{aligned}
& ||\left|\boldsymbol{x}_{n+1}-\boldsymbol{x}\right|\left\||=|\left|\boldsymbol{\Lambda}_{n}\left(\boldsymbol{x}_{n}-\boldsymbol{x}\right)+\left(\mathbf{I d}-\boldsymbol{\Lambda}_{n}\right)\left(J_{\gamma_{n} \boldsymbol{A}_{n}}\left(\boldsymbol{x}_{n}-\gamma_{n}\left(\boldsymbol{B}_{n} \boldsymbol{x}_{n}+\boldsymbol{d}_{n}\right)\right)-\boldsymbol{x}+\boldsymbol{c}_{n}\right) \|\right|\right. \\
& \leq||\left|\boldsymbol{\Lambda}_{n}\right|||||\left|\boldsymbol{x}_{n}-\boldsymbol{x}\right|||+||\left|\mathbf{I d}-\boldsymbol{\Lambda}_{n}\right|||||\left|\boldsymbol{c}_{n}\right||| \\
& +||\left|\mathbf{I d}-\boldsymbol{\Lambda}_{n}\right|||||\left|J_{\gamma_{n}} \boldsymbol{A}_{n}\left(\boldsymbol{x}_{n}-\gamma_{n}\left(\boldsymbol{B}_{n} \boldsymbol{x}_{n}+\boldsymbol{d}_{n}\right)\right)-\boldsymbol{x}\right| \| \\
& \leq||\left|\boldsymbol{\Lambda}_{n}\right|||||\left|\boldsymbol{x}_{n}-\boldsymbol{x}\right|||+||\left|\mathbf{I d}-\boldsymbol{\Lambda}_{n}\right|||||\left|\boldsymbol{c}_{n}\right||| \\
& +||\left|\mathbf{I d}-\boldsymbol{\Lambda}_{n}\right|||\left(\left(1+2 \beta \kappa_{n}\right)||\left|\boldsymbol{x}_{n}-\boldsymbol{x}\left\|\left|+2 \beta \kappa_{n}\right|\right\| \boldsymbol{x}-\boldsymbol{z}\right|||\right. \\
& \left.+2 \beta||\left|\boldsymbol{d}_{n}\right|||+||\left|\boldsymbol{e}_{n}\right|||\right) \\
& \leq\left(||\left|\boldsymbol{\Lambda}_{n}\right|||+||\left|\mathbf{I d}-\boldsymbol{\Lambda}_{n}\right|||\right)||\left|\boldsymbol{x}_{n}-\boldsymbol{x}\right|||+||\left|\mathbf{I d}-\boldsymbol{\Lambda}_{n}\right|||\left(||\left|\boldsymbol{c}_{n}\right|||+2 \beta \kappa_{n}||\left|\boldsymbol{x}_{n}-\boldsymbol{x}\right|||\right. \\
& \left.+2 \beta \kappa_{n}|||\boldsymbol{x}-\boldsymbol{z}|||+2 \beta||\left|\boldsymbol{d}_{n}\right|||+||\left|\boldsymbol{e}_{n}\right|||\right) \\
& \leq\left(1+\tau_{n}\right)\left|\left\|\boldsymbol{x}_{n}-\boldsymbol{x}\right\|\right|+\left|\left\|\boldsymbol { c } _ { n } \left|\left\|+2 \beta \kappa_{n}\left|\left\|\boldsymbol{x}_{n}-\boldsymbol{x} \mid\right\|\right.\right.\right.\right.\right. \\
& +2 \beta \kappa_{n}|||\boldsymbol{x}-\boldsymbol{z}|||+2 \beta\left|\| \boldsymbol{d}_{n}\right|||+|| \boldsymbol{e}_{n}|| \mid \\
& \leq\left(1+\alpha_{n}\right)\left|\left\|\boldsymbol{x}_{n}-\boldsymbol{x}\right\|\right|+\delta_{n}
\end{aligned}
$$

where

$$
\alpha_{n}=\tau_{n}+2 \beta \kappa_{n} \quad \text { and } \quad \delta_{n}=\left\|\left|\boldsymbol{c}_{n}\right|\right\|+2 \beta \kappa_{n}\left|\left\|\boldsymbol{x}-\boldsymbol{z}\left|\left\|+2 \beta\left|\left\|\boldsymbol { d } _ { n } \left|\left\|+\left|\left\|\boldsymbol{e}_{n}\right\|\right|\right.\right.\right.\right.\right.\right.\right.\right.
$$


In turn, it follows from (2.32), (2.35), (2.26), (2.27), and (2.41) that $\sum_{k \in \mathbb{N}} \alpha_{k}<+\infty$ and $\sum_{k \in \mathbb{N}} \delta_{k}<$ $+\infty$. Thus, (2.44) and [39, Lemma 2.2.2] yield

$$
\sup _{k \in \mathbb{N}}\left\|\left|\boldsymbol{x}_{k}-\boldsymbol{x} \|\right|<+\infty\right.
$$

and, using (2.35) and (2.27), we derive from (2.37) that

$$
\sum_{k \in \mathbb{N}}||\left|\boldsymbol{b}_{k}\right| \|<+\infty
$$

In view of (2.36) and (2.38), (2.33) is equivalent to

$$
\boldsymbol{x}_{n+1}=\boldsymbol{\Lambda}_{n} \boldsymbol{x}_{n}+\left(\mathbf{I d}-\boldsymbol{\Lambda}_{n}\right)\left(J_{\gamma_{n}} \boldsymbol{A}\left(\boldsymbol{T}_{n} \boldsymbol{x}_{n}-\gamma_{n} \boldsymbol{b}_{n}\right)+\boldsymbol{h}_{n}\right),
$$

where

$$
\boldsymbol{h}_{n}=J_{\gamma_{n} \boldsymbol{A}_{n}}\left(\boldsymbol{T}_{n} \boldsymbol{x}_{n}-\gamma_{n} \boldsymbol{b}_{n}\right)-J_{\gamma_{n} \boldsymbol{A}}\left(\boldsymbol{T}_{n} \boldsymbol{x}_{n}-\gamma_{n} \boldsymbol{b}_{n}\right)+\boldsymbol{c}_{n} .
$$

Now set $\mu=\sup _{k \in \mathbb{N}}\left|\left\|\boldsymbol{x}_{k}-\boldsymbol{x}\left|\left\|+\rho+2 \beta \sup _{k \in \mathbb{N}}|| \boldsymbol{b}_{k}\right\|\right|\right.\right.$. Then it follows from (2.46), and (2.47) that $\mu<+\infty$. Moreover, we deduce from the nonexpansivity of $\boldsymbol{T}_{n}$ and (2.39) that

$$
\begin{aligned}
\left\|\boldsymbol{T}_{n} \boldsymbol{x}_{n}-\gamma_{n} \boldsymbol{b}_{n} \mid\right\| & \leq\left\|\left|\boldsymbol{T}_{n} \boldsymbol{x}_{n}-\boldsymbol{T}_{n} \boldsymbol{x}\left\|\left|+\left\|\left|\boldsymbol{T}_{n} \boldsymbol{x}\right|\right\|+2 \beta\|\| \boldsymbol{b}_{n}\right|\right\|\right|\right. \\
& \leq\|\| \boldsymbol{x}_{n}-\boldsymbol{x}\left\|\left|+\rho+2 \beta\|\| \boldsymbol{b}_{n} \|\right|\right. \\
& \leq \mu
\end{aligned}
$$

Hence, appealing to (2.21) and (2.26), we infer from (2.49) that

$$
\sum_{k \in \mathbb{N}}\left\|\left|\boldsymbol{h}_{k}\right|\right\|<+\infty
$$

Note that, upon introducing

$$
\boldsymbol{a}_{n}=\boldsymbol{h}_{n}+\frac{1}{1-\lambda_{n}}\left(\boldsymbol{\Lambda}_{n}-\lambda_{n} \mathbf{I d}\right)\left(\boldsymbol{x}_{n}-J_{\gamma_{n}} \boldsymbol{A}\left(\boldsymbol{T}_{n} \boldsymbol{x}_{n}-\gamma_{n} \boldsymbol{b}_{n}\right)-\boldsymbol{h}_{n}\right),
$$

we can rewrite (2.48) in the equivalent form (2.11), namely

$$
\boldsymbol{x}_{n+1}=\lambda_{n} \boldsymbol{x}_{n}+\left(1-\lambda_{n}\right)\left(J_{\gamma_{n}} \boldsymbol{A}\left(\boldsymbol{x}_{n}-\gamma_{n}\left(\boldsymbol{B} \boldsymbol{x}_{n}+\boldsymbol{b}_{n}\right)\right)+\boldsymbol{a}_{n}\right) .
$$

Using (2.40) and the nonexpansivity of $J_{\gamma_{n} \boldsymbol{A}}$ and $\boldsymbol{T}_{n}$, we get

$$
\begin{aligned}
\left\|\left|\boldsymbol{x}_{n}-J_{\gamma_{n} \boldsymbol{A}}\left(\boldsymbol{T}_{n} \boldsymbol{x}_{n}-\gamma_{n} \boldsymbol{b}_{n}\right)-\boldsymbol{h}_{n}\right|\right\| \mid \leq & \left\|\left|\boldsymbol{x}_{n}-\boldsymbol{x}\left\|\left|+\left\|J_{\gamma_{n} \boldsymbol{A}}\left(\boldsymbol{T}_{n} \boldsymbol{x}\right)-J_{\gamma_{n} \boldsymbol{A}}\left(\boldsymbol{T}_{n} \boldsymbol{x}_{n}-\gamma_{n} \boldsymbol{b}_{n}\right) \mid\right\|\right.\right.\right.\right. \\
& +\left\|\left|\boldsymbol{h}_{n} \|\right|\right. \\
\leq & 2\left|\left\|\boldsymbol{x}_{n}-\boldsymbol{x}\right\|\right|+2 \beta\left|\left\|\boldsymbol{b}_{n}\right\|\right|+\left\|\boldsymbol{h}_{n} \mid\right\| .
\end{aligned}
$$

Therefore, we derive from (2.46), (2.47), and (2.51) that

$$
\nu=\sup _{k \in \mathbb{N}}\left\|\boldsymbol{x}_{k}-J_{\gamma_{k} \boldsymbol{A}}\left(\boldsymbol{T}_{k} \boldsymbol{x}_{k}-\gamma_{k} \boldsymbol{b}_{k}\right)-\boldsymbol{h}_{k}\right\| \mid<+\infty,
$$

and hence, from (2.52) and the inequality $\lambda_{n} \leq 1-\varepsilon$, that

$$
\begin{aligned}
\left\|\left|\boldsymbol{a}_{n}\right|\right\| & \leq\left\|\left|\boldsymbol{h}_{n}\right|\right\|+\frac{1}{1-\lambda_{n}}|| \boldsymbol{\Lambda}_{n}-\lambda_{n} \mathbf{I d}\left|\left\|\left|\left\|\boldsymbol{x}_{n}-J_{\gamma_{n} \boldsymbol{A}}\left(\boldsymbol{T}_{n} \boldsymbol{x}_{n}-\gamma_{n} \boldsymbol{b}_{n}\right)-\boldsymbol{h}_{n}\right\|\right|\right.\right. \\
& \leq||\left|\boldsymbol{h}_{n}\right| \|+\frac{\nu}{\varepsilon} \max _{1 \leq i \leq m}\left|\lambda_{i, n}-\lambda_{n}\right| .
\end{aligned}
$$


Thus, using (2.51) and arguing as in (2.32), we get

$$
\sum_{k \in \mathbb{N}}\left|\left\|\boldsymbol{a}_{k} \mid\right\|<+\infty\right.
$$

However, Theorem 2.8(i) asserts that, under (2.19), (2.24), (2.47), (2.57), and the hypotheses on $\left(\gamma_{n}\right)_{n \in \mathbb{N}}$ and $\left(\lambda_{n}\right)_{n \in \mathbb{N}}$ in Algorithm 2.2, the sequence $\left(\boldsymbol{x}_{n}\right)_{n \in \mathbb{N}}$ generated by (2.53) converges weakly to a point in $\boldsymbol{Z}$. Since (2.53) is equivalent to (2.3) and (2.23) is equivalent to (1.7), the proof is complete.

We conclude this section with the following theorem, in which we describe instances of strong convergence derived from Theorem 2.8.

Theorem 2.10 Let $\left(\left(x_{i, n}\right)_{n \in \mathbb{N}}\right)_{1 \leq i \leq m}$ and $\left(x_{i}\right)_{1 \leq i \leq m}$ be as in Theorem 2.9. Then the following hold.

(i) Suppose that, for some $i \in\{1, \ldots, m\}, A_{i}$ is demiregular at $x_{i}$ (see Proposition 2.4 for special cases). Then $x_{i, n} \rightarrow x_{i}$.

(ii) Suppose that the operator $\left(y_{j}\right)_{1 \leq j \leq m} \mapsto\left(B_{i}\left(y_{j}\right)_{1 \leq j \leq m}\right)_{1 \leq i \leq m}$ is demiregular at $\left(x_{i}\right)_{1 \leq i \leq m}$ (see Proposition 2.4 for special cases). Then, for every $i \in\{1, \ldots, m\}, x_{i, n} \rightarrow x_{i}$.

(iii) Suppose that the set of solutions to Problem 1.1 has a nonempty interior. Then, for every $i \in\{1, \ldots, m\}, x_{i, n} \rightarrow x_{i}$.

Proof. We use the same product space setting and notation as in the proof of Theorem 2.9. In particular, we set $\boldsymbol{x}=\left(x_{1}, \ldots, x_{m}\right)$ and $\mathcal{H}=\mathcal{H}_{1} \oplus \cdots \oplus \mathcal{H}_{m}$, and we define

$$
\boldsymbol{A}: \mathcal{H} \rightarrow 2^{\mathcal{H}}: \boldsymbol{y} \mapsto \underset{i=1}{\times} A_{i} y_{i} \quad \text { and } \quad \boldsymbol{B}: \mathcal{H} \rightarrow \mathcal{H}: \boldsymbol{y} \mapsto\left(B_{i} \boldsymbol{y}\right)_{1 \leq i \leq m}
$$

(i): Set $v_{i}=-B_{i}\left(x_{1}, \ldots, x_{m}\right)$ and

$$
(\forall n \in \mathbb{N}) \quad\left\{\begin{array}{l}
y_{i, n}=J_{\gamma_{n} A_{i}}\left(x_{i, n}-\gamma_{n} B_{i}\left(x_{1, n}, \ldots, x_{m, n}\right)\right) \\
v_{i, n}=\gamma_{n}^{-1}\left(x_{i, n}-y_{i, n}\right)-B_{i}\left(x_{1, n}, \ldots, x_{m, n}\right) .
\end{array}\right.
$$

It follows from Theorem 2.8(i) that

$$
x_{i, n} \rightarrow x_{i}
$$

from Theorem 2.8(ii) that

$$
\left\|B_{i}\left(x_{1, n}, \ldots, x_{m, n}\right)-B_{i}\left(x_{1}, \ldots, x_{m}\right)\right\|=\left\|B_{i} \boldsymbol{x}_{n}-B_{i} \boldsymbol{x}\right\| \leq\left\|\boldsymbol{B} \boldsymbol{x}_{n}-\boldsymbol{B} \boldsymbol{x}\right\| \| \rightarrow 0,
$$

and from Theorem 2.8(iii) and (2.20) that

$$
\left\|x_{i, n}-y_{i, n}\right\| \leq\left\|\boldsymbol{x}_{n}-J_{\gamma_{n} \boldsymbol{A}}\left(\boldsymbol{x}_{n}-\gamma_{n} \boldsymbol{B} \boldsymbol{x}_{n}\right)\right\| \| \rightarrow 0 .
$$

Combining (2.60) and (2.62), we obtain

$$
y_{i, n} \rightarrow x_{i}
$$

Next, we derive from (2.59) that

$$
(\forall n \in \mathbb{N}) \quad\left(y_{i, n}, v_{i, n}\right) \in \operatorname{gra} A_{i}
$$


and that

$$
(\forall n \in \mathbb{N}) \quad\left\|v_{i, n}-v_{i}\right\| \leq \frac{\left\|x_{i, n}-y_{i, n}\right\|}{\gamma_{n}}+\left\|B_{i}\left(x_{1, n}, \ldots, x_{m, n}\right)-B_{i}\left(x_{1}, \ldots, x_{m}\right)\right\| .
$$

Hence, it follows from (2.62), the condition $\inf _{n \in \mathbb{N}} \gamma_{n}>0$, and (2.61), that

$$
v_{i, n} \rightarrow v_{i} \text {. }
$$

Altogether, (2.63), (2.64), (2.66), and Definition 2.3 yield $y_{i, n} \rightarrow x_{i}$. In turn, appealing to (2.62), we conclude that $x_{i, n} \rightarrow x_{i}$.

(ii)\&(iii): As seen in the proof of Theorem 2.9, the convergence properties of $\left(\boldsymbol{x}_{n}\right)_{n \in \mathbb{N}}=$ $\left(\left(x_{i, n}\right)_{n \in \mathbb{N}}\right)_{1 \leq i \leq m}$ follow from those listed in Theorem 2.8 and applied to the operators defined in (2.58); moreover, the set of solutions to Problem 1.1 is $\boldsymbol{Z}=(\boldsymbol{A}+\boldsymbol{B})^{-1}(\mathbf{0})$. Therefore, (ii) follows from Theorem 2.8(iv)(b), and (iii) from Theorem 2.8(iv)(c).

\section{Coupling evolution inclusions}

Evolution inclusions arise in various fields of applied mathematics [30, 42]. In this section, we address the problem of solving systems of coupled evolution inclusions with periodicity conditions.

Let us recall some standard notation $[15,48]$. Fix $T \in] 0,+\infty[$ and $p \in[1,+\infty[$. Then $\mathcal{D}(] 0, T[)$ is the set of infinitely differentiable functions from $] 0, T[$ to $\mathbb{R}$ with compact support in $] 0, T[$. Given a real Hilbert space $\mathrm{H}, \mathcal{C}([0, T] ; \mathrm{H})$ is the space of continuous functions from $[0, T]$ to $\mathrm{H}$ and $L^{p}([0, T] ; \mathrm{H})$ is the space of classes of equivalences of Borel measurable functions $x:[0, T] \rightarrow$ $\mathrm{H}$ such that $\int_{0}^{T}\|x(t)\|_{\mathrm{H}}^{p} d t<+\infty . L^{2}([0, T] ; \mathrm{H})$ is a Hilbert space with scalar product $(x, y) \mapsto$ $\int_{0}^{T}\langle x(t) \mid y(t)\rangle_{\mathrm{H}} d t$. Now take $x$ and $y$ in $L^{1}([0, T] ; \mathrm{H})$. Then $y$ is the weak derivative of $x$ if $\int_{0}^{T} \phi(t) y(t) d t=-\int_{0}^{T}(d \phi(t) / d t) x(t) d t$ for every $\phi \in \mathcal{D}(] 0, T[)$, in which case we use the notation $y=x^{\prime}$. Moreover,

$$
W^{1,2}([0, T] ; \mathbf{H})=\left\{x \in L^{2}([0, T] ; \mathbf{H}) \mid x^{\prime} \in L^{2}([0, T] ; \mathbf{H})\right\},
$$

equipped with the scalar product $(x, y) \mapsto \int_{0}^{T}\langle x(t) \mid y(t)\rangle_{\mathrm{H}} d t+\int_{0}^{T}\left\langle x^{\prime}(t) \mid y^{\prime}(t)\right\rangle_{\mathrm{H}} d t$, is a Hilbert space.

Problem 3.1 Let $\left(\mathrm{H}_{i}\right)_{1 \leq i \leq m}$ be real Hilbert spaces and let $\left.T \in\right] 0,+\infty[$. For every $i \in\{1, \ldots, m\}$, set

$$
\mathcal{W}_{i}=\left\{x \in \mathcal{C}\left([0, T] ; \mathrm{H}_{i}\right) \cap W^{1,2}\left([0, T] ; \mathrm{H}_{i}\right) \mid x(T)=x(0)\right\},
$$

let $\mathrm{f}_{i} \in \Gamma_{0}\left(\mathrm{H}_{i}\right)$, and let $\mathrm{B}_{i}: \mathrm{H}_{1} \times \cdots \times \mathrm{H}_{m} \rightarrow \mathrm{H}_{i}$. It is assumed that there exists $\left.\beta \in\right] 0,+\infty[$ such that

$$
\begin{aligned}
& \left(\forall\left(\mathrm{x}_{1}, \ldots, \mathrm{x}_{m}\right) \in \mathrm{H}_{1} \times \cdots \times \mathrm{H}_{m}\right)\left(\forall\left(\mathrm{y}_{1}, \ldots, \mathrm{y}_{m}\right) \in \mathrm{H}_{1} \times \cdots \times \mathrm{H}_{m}\right) \\
& \sum_{i=1}^{m}\left\langle\mathrm{~B}_{i}\left(\mathrm{x}_{1}, \ldots, \mathrm{x}_{m}\right)-\mathrm{B}_{i}\left(\mathrm{y}_{1}, \ldots, \mathrm{y}_{m}\right) \mid \mathrm{x}_{i}-\mathrm{y}_{i}\right\rangle_{\mathrm{H}_{i}} \geq \beta \sum_{i=1}^{m}\left\|\mathrm{~B}_{i}\left(\mathrm{x}_{1}, \ldots, \mathrm{x}_{m}\right)-\mathrm{B}_{i}\left(\mathrm{y}_{1}, \ldots, \mathrm{y}_{m}\right)\right\|_{\mathrm{H}_{i}}^{2} .
\end{aligned}
$$

The problem is to

find $x_{1} \in \mathcal{W}_{1}, \ldots, x_{m} \in \mathcal{W}_{m}$ such that

$$
\left.(\forall i \in\{1, \ldots, m\}) \quad 0 \in x_{i}^{\prime}(t)+\partial \mathrm{f}_{i}\left(x_{i}(t)\right)+\mathrm{B}_{i}\left(x_{1}(t), \ldots, x_{m}(t)\right) \text { a.e. on }\right] 0, T[,
$$

under the assumption that such solutions exist. 
Algorithm 3.2 Fix $\varepsilon \in] 0, \min \{1, \beta\}\left[,\left(\gamma_{n}\right)_{n \in \mathbb{N}}\right.$ in $[\varepsilon, 2 \beta-\varepsilon]$, and $\left(\lambda_{n}\right)_{n \in \mathbb{N}}$ in $[0,1-\varepsilon]$. Let, for every $n \in \mathbb{N}$ and every $i \in\{1, \ldots, m\}, y_{i, n}$ be the unique solution in $\mathcal{W}_{i}$ to the inclusion

$$
\begin{aligned}
\frac{x_{i, n}(t)-y_{i, n}(t)}{\gamma_{n}}-\left(\mathrm{B}_{i}\left(x_{1, n}(t), \ldots, x_{m, n}(t)\right)+\right. & \left.b_{i, n}(t)\right) \\
& \left.\in y_{i, n}^{\prime}(t)+\partial \mathrm{f}_{i}\left(y_{i, n}(t)\right)+e_{i, n}(t) \text { a.e. on }\right] 0, T[
\end{aligned}
$$

and set

$$
x_{i, n+1}=\lambda_{i, n} x_{i, n}+\left(1-\lambda_{i, n}\right) y_{i, n}
$$

where, for every $i \in\{1, \ldots, m\}$, the following hold.

(i) $x_{i, 0} \in W^{1,2}\left([0, T] ; \mathrm{H}_{i}\right)$.

(ii) $\left(b_{i, n}\right)_{n \in \mathbb{N}}$ and $\left(e_{i, n}\right)_{n \in \mathbb{N}}$ are sequences in $L^{2}\left([0, T] ; \mathrm{H}_{i}\right)$ such that

$$
\sum_{n \in \mathbb{N}} \sqrt{\int_{0}^{T}\left\|b_{i, n}(t)\right\|_{\mathrm{H}_{i}}^{2} d t}<+\infty \text { and } \sum_{n \in \mathbb{N}} \sqrt{\int_{0}^{T}\left\|e_{i, n}(t)\right\|_{\mathrm{H}_{i}}^{2} d t}<+\infty .
$$

(iii) $\left(\lambda_{i, n}\right)_{n \in \mathbb{N}}$ is a sequence in $\left[0,1\left[\right.\right.$ such that $\sum_{n \in \mathbb{N}}\left|\lambda_{i, n}-\lambda_{n}\right|<+\infty$.

In (3.5), $b_{i, n}(t)$ models the error tolerated in the computation of $\mathrm{B}_{i}\left(x_{1, n}(t), \ldots, x_{m, n}(t)\right)$, while $e_{i, n}(t)$ models the error tolerated in solving the inclusion with respect to $\partial \mathrm{f}_{i}\left(y_{i, n}(t)\right)$.

We now examine the weak convergence properties of Algorithm 3.2 (strong convergence conditions can be derived from Theorem 2.10).

Theorem 3.3 Let $\left(\left(x_{i, n}\right)_{n \in \mathbb{N}}\right)_{1 \leq i \leq m}$ be sequences generated by Algorithm 3.2. Then, for every $i \in$ $\{1, \ldots, m\},\left(x_{i, n}\right)_{n \in \mathbb{N}}$ converges weakly in $W^{1,2}\left([0, T] ; \mathrm{H}_{i}\right)$ to a point $x_{i} \in \mathcal{W}_{i}$, and $\left(x_{i}\right)_{1 \leq i \leq m}$ is a solution to Problem 3.1.

Proof. For every $i \in\{1, \ldots, m\}$, set $\mathcal{H}_{i}=L^{2}\left([0, T] ; \mathrm{H}_{i}\right)$ and

$$
\begin{aligned}
& A_{i}: \mathcal{H}_{i} \rightarrow 2^{\mathcal{H}_{i}}
\end{aligned}
$$

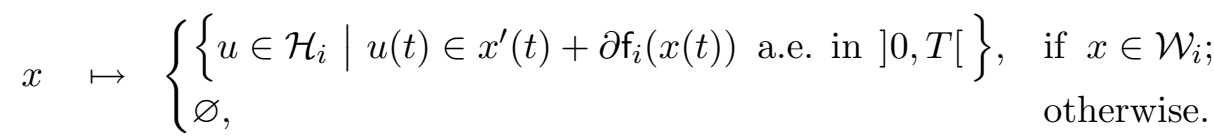

Let us first show that the operators $\left(A_{i}\right)_{1 \leq i \leq m}$ are maximal monotone. For this purpose, let $i \in$ $\{1, \ldots, m\},(x, u) \in \operatorname{gra} A_{i}$, and $(y, v) \in \operatorname{gra} A_{i}$. It follows from (3.8) that, almost everywhere on ] $0, T\left[, u(t)-x^{\prime}(t) \in \partial \mathrm{f}_{i}(x(t))\right.$ and $v(t)-y^{\prime}(t) \in \partial \mathrm{f}_{i}(y(t))$. Therefore, by monotonicity of $\partial \mathrm{f}_{i}$, we have

$$
\int_{0}^{T}\left\langle x(t)-y(t) \mid\left(u(t)-x^{\prime}(t)\right)-\left(v(t)-y^{\prime}(t)\right)\right\rangle_{\mathbf{H}_{i}} d t \geq 0 .
$$


Hence,

$$
\begin{aligned}
\langle x-y \mid u-v\rangle= & \int_{0}^{T}\langle x(t)-y(t) \mid u(t)-v(t)\rangle_{\mathrm{H}_{i}} d t \\
= & \int_{0}^{T}\left\langle x(t)-y(t) \mid\left(u(t)-x^{\prime}(t)\right)-\left(v(t)-y^{\prime}(t)\right)\right\rangle_{\mathrm{H}_{i}} d t \\
& +\int_{0}^{T}\left\langle x(t)-y(t) \mid x^{\prime}(t)-y^{\prime}(t)\right\rangle_{\mathrm{H}_{i}} d t \\
\geq & \frac{1}{2} \int_{0}^{T} \frac{d\|x(t)-y(t)\|_{\mathrm{H}_{i}}^{2}}{d t} d t \\
= & \frac{1}{2}\left(\|x(T)-y(T)\|_{\mathrm{H}_{i}}^{2}-\|x(0)-y(0)\|_{\mathrm{H}_{i}}^{2}\right) \\
= & 0 .
\end{aligned}
$$

Thus, $A_{i}$ is monotone. To prove maximality, set $\mathrm{g}_{i}=(1 / 2)\|\cdot\|_{\mathrm{H}_{i}}^{2}+\mathrm{f}_{i}$. Then $\mathrm{g}_{i} \in \Gamma_{0}\left(\mathrm{H}_{i}\right)$ and $\partial \mathrm{g}_{i}=\mathrm{Id}+\partial \mathrm{f}_{i}$. Moreover, since $\mathrm{f}_{i} \in \Gamma_{0}\left(\mathrm{H}_{i}\right)$, it follows from the Fenchel-Moreau theorem that it is minorized by a continuous affine functional, say $\mathrm{f}_{i} \geq\langle\cdot \mid \mathrm{v}\rangle_{\mathrm{H}_{i}}+\eta$ for some $\mathrm{v} \in \mathrm{H}_{i}$ and $\eta \in \mathbb{R}$. Now, let $\mathrm{y} \in \operatorname{dom}_{i}=\operatorname{dom}_{i}$ and take $(\mathrm{x}, \mathrm{u}) \in \operatorname{gra} \partial \mathrm{g}_{i}$. Then (1.10) and Cauchy-Schwarz imply the coercivity property

$$
\begin{aligned}
\frac{\langle\mathrm{x}-\mathrm{y} \mid \mathrm{u}\rangle_{\mathrm{H}_{i}}}{\|\mathrm{x}\|_{\mathrm{H}_{i}}} & \geq \frac{\mathrm{g}_{i}(\mathrm{x})-\mathrm{g}_{i}(\mathrm{y})}{\|\mathrm{x}\|_{\mathrm{H}_{i}}} \\
& =\frac{\|\mathrm{x}\|_{\mathrm{H}_{i}}}{2}+\frac{\mathrm{f}_{i}(\mathrm{x})-\mathrm{g}_{i}(\mathrm{y})}{\|\mathrm{x}\|_{\mathrm{H}_{i}}} \\
& \geq \frac{\|\mathrm{x}\|_{\mathrm{H}_{i}}}{2}-\|\mathrm{v}\|_{\mathrm{H}_{i}}+\frac{\eta-\mathrm{g}_{i}(\mathrm{y})}{\|\mathrm{x}\|_{\mathrm{H}_{i}}} \\
& \rightarrow+\infty \text { as }\|\mathrm{x}\|_{\mathrm{H}_{i}} \rightarrow+\infty
\end{aligned}
$$

Therefore, [15, Corollaire 3.4] asserts that for every $w \in \mathcal{H}_{i}$ there exists $z \in \mathcal{W}_{i}$ such that

$$
\left.w(t) \in z^{\prime}(t)+\partial \mathrm{g}_{i}(z(t))=z^{\prime}(t)+z(t)+\partial \mathrm{f}_{i}(z(t)) \text { a.e. on }\right] 0, T[,
$$

i.e., by (3.8), such that $w-z \in A_{i} z$. This shows that the range of $\mathrm{Id}+A_{i}$ is $\mathcal{H}_{i}$ and hence, by Minty's theorem [5, Theorem 3.5.8], that $A_{i}$ is maximal monotone.

Next, for every $i \in\{1, \ldots, m\}$ and every $\left(x_{1}, \ldots, x_{m}\right) \in \mathcal{H}_{1} \times \cdots \times \mathcal{H}_{m}$, define almost everywhere

$$
B_{i}\left(x_{1}, \ldots, x_{m}\right):[0, T] \rightarrow \mathrm{H}_{i}: t \mapsto \mathrm{B}_{i}\left(x_{1}(t), \ldots, x_{m}(t)\right) .
$$

Now let $\left(x_{1}, \ldots, x_{m}\right) \in \mathcal{H}_{1} \times \cdots \times \mathcal{H}_{m}$ and set $(\forall i \in\{1, \ldots, m\}) \mathbf{b}_{i}=\mathrm{B}_{i}(0, \ldots, 0)$. Then it follows from (3.3) and Cauchy-Schwarz that, almost everywhere on $[0, T]$,

$$
\begin{aligned}
\beta \sum_{j=1}^{m}\left\|\mathrm{~B}_{j}\left(x_{1}(t), \ldots, x_{m}(t)\right)-\mathrm{b}_{j}\right\|_{\mathrm{H}_{j}}^{2} & \leq \sum_{j=1}^{m}\left\langle\mathrm{~B}_{j}\left(x_{1}(t), \ldots, x_{m}(t)\right)-\mathrm{b}_{j} \mid x_{j}(t)-0\right\rangle_{\mathrm{H}_{j}} \\
& \leq \sum_{j=1}^{m}\left\|\mathrm{~B}_{j}\left(x_{1}(t), \ldots, x_{m}(t)\right)-\mathrm{b}_{j}\right\|_{\mathrm{H}_{j}}\left\|x_{j}(t)\right\|_{\mathrm{H}_{j}} \\
& \leq \sqrt{\sum_{j=1}^{m}\left\|\mathrm{~B}_{j}\left(x_{1}(t), \ldots, x_{m}(t)\right)-\mathrm{b}_{j}\right\|_{\mathrm{H}_{j}}^{2}} \sqrt{\sum_{j=1}^{m}\left\|x_{j}(t)\right\|_{\mathrm{H}_{j}}^{2}} .
\end{aligned}
$$


Therefore, for every $i \in\{1, \ldots, m\}$,

$$
\begin{aligned}
\left\|B_{i}\left(x_{1}, \ldots, x_{m}\right)(t)\right\|_{\mathrm{H}_{i}}^{2} & \leq 2\left(\left\|\mathrm{~b}_{i}\right\|_{\mathrm{H}_{i}}^{2}+\left\|B_{i}\left(x_{1}, \ldots, x_{m}\right)(t)-\mathrm{b}_{i}\right\|_{\mathrm{H}_{i}}^{2}\right) \\
& \leq 2\left(\left\|\mathrm{~b}_{i}\right\|_{\mathrm{H}_{i}}^{2}+\sum_{j=1}^{m}\left\|\mathrm{~B}_{j}\left(x_{1}(t), \ldots, x_{m}(t)\right)-\mathrm{b}_{j}\right\|_{\mathrm{H}_{j}}^{2}\right) \\
& \left.\leq 2\left(\left\|\mathrm{~b}_{i}\right\|_{\mathrm{H}_{i}}^{2}+\frac{1}{\beta^{2}} \sum_{j=1}^{m}\left\|x_{j}(t)\right\|_{\mathrm{H}_{j}}^{2}\right) \text { a.e. on }\right] 0, T[,
\end{aligned}
$$

which yields

$$
\int_{0}^{T}\left\|B_{i}\left(x_{1}, \ldots, x_{m}\right)(t)\right\|_{\mathrm{H}_{i}}^{2} d t \leq 2 T\left\|\mathrm{~b}_{i}\right\|_{\mathrm{H}_{i}}^{2}+\frac{2}{\beta^{2}} \sum_{j=1}^{m}\left\|x_{j}\right\|^{2}
$$

so that we can now claim that $B_{i}: \mathcal{H}_{1} \times \cdots \times \mathcal{H}_{m} \rightarrow L^{2}\left([0, T] ; \mathrm{H}_{i}\right)=\mathcal{H}_{i}$. In addition, upon integrating, we derive from (3.3) and (3.13) that, for every $\left(y_{1}, \ldots, y_{m}\right) \in \mathcal{H}_{1} \times \cdots \times \mathcal{H}_{m}$,

$$
\sum_{i=1}^{m}\left\langle B_{i}\left(x_{1}, \ldots, x_{m}\right)-B_{i}\left(y_{1}, \ldots, y_{m}\right) \mid x_{i}-y_{i}\right\rangle \geq \beta \sum_{i=1}^{m}\left\|B_{i}\left(x_{1}, \ldots, x_{m}\right)-B_{i}\left(y_{1}, \ldots, y_{m}\right)\right\|^{2} .
$$

We have thus established (1.6).

Let us now make the connection between Algorithm 3.2 and Algorithm 2.2. For every $n \in \mathbb{N}$ and every $i \in\{1, \ldots, m\}$, it follows from (3.5), (3.8), (3.13), and the maximal monotonicity of $A_{i}$ that $y_{i, n}$ is uniquely defined and can be expressed as

$$
y_{i, n}=J_{\gamma_{n} A_{i}}\left(x_{i, n}-\gamma_{n}\left(B_{i}\left(x_{1, n}, \ldots, x_{m, n}\right)+b_{i, n}\right)\right)+a_{i, n}
$$

where

$$
\begin{aligned}
a_{i, n}=J_{\gamma_{n} A_{i}}\left(-\gamma_{n} e_{i, n}+x_{i, n}-\gamma_{n}\left(B_{i}\left(x_{1, n}, \ldots, x_{m, n}\right)+b_{i, n}\right)\right) & \\
& -J_{\gamma_{n} A_{i}}\left(x_{i, n}-\gamma_{n}\left(B_{i}\left(x_{1, n}, \ldots, x_{m, n}\right)+b_{i, n}\right)\right)
\end{aligned}
$$

and we therefore deduce from (3.5) and (3.6) that

$$
x_{i, n+1}=\lambda_{i, n} x_{i, n}+\left(1-\lambda_{i, n}\right)\left(J_{\gamma_{n} A_{i}}\left(x_{i, n}-\gamma_{n}\left(B_{i}\left(x_{1, n}, \ldots, x_{m, n}\right)+b_{i, n}\right)\right)+a_{i, n}\right) .
$$

We observe that (3.20) derives from (2.3), where $A_{i, n} \equiv A_{i}$ and $B_{i, n} \equiv B_{i}$. On the other hand, for every $i \in\{1, \ldots, m\}$, by nonexpansivity of the operators $\left(J_{\gamma_{n} A_{i}}\right)_{n \in \mathbb{N}}$, we deduce from (3.19) and (3.7) that

$$
\sum_{n \in \mathbb{N}}\left\|a_{i, n}\right\| \leq \sum_{n \in \mathbb{N}} \gamma_{n}\left\|e_{i, n}\right\| \leq 2 \beta \sum_{n \in \mathbb{N}}\left\|e_{i, n}\right\|<+\infty .
$$

As a result, all the hypotheses of Algorithm 2.2 are satisfied and hence Theorem 2.9 asserts that, for every $i \in\{1, \ldots, m\},\left(x_{i, n}\right)_{n \in \mathbb{N}}$ converges weakly in $\mathcal{H}_{i}=L^{2}\left([0, T] ; \mathrm{H}_{i}\right)$ to a point $x_{i}$, and $\left(x_{i}\right)_{1 \leq i \leq m}$ satisfies

$$
(\forall i \in\{1, \ldots, m\}) \quad 0 \in A_{i} x_{i}+B_{i}\left(x_{1}, \ldots, x_{m}\right) .
$$

Accordingly,

$$
\sigma=\max _{1 \leq i \leq m} \sup _{n \in \mathbb{N}}\left\|x_{i, n}\right\|<+\infty
$$


and $(\forall i \in\{1, \ldots, m\}) x_{i} \in \operatorname{dom} A_{i} \subset \mathcal{W}_{i}$. Moreover since, in view of (3.8) and (3.13), (3.22) reduces to (3.4), $\left(x_{i}\right)_{1 \leq i \leq m}$ is a solution to Problem 3.1.

To complete the proof, let $i \in\{1, \ldots, m\}$. To show that $\left(x_{i, n}\right)_{n \in \mathbb{N}}$ converges weakly to $x_{i}$ in $W^{1,2}\left([0, T] ; \mathrm{H}_{i}\right)$, it remains to show that $\left(x_{i, n}^{\prime}\right)_{n \in \mathbb{N}}$ converges weakly to $x_{i}^{\prime}$ in $L^{2}\left([0, T] ; \mathrm{H}_{i}\right)$. We first observe that $\left(x_{i, n}\right)_{n \in \mathbb{N}}$ lies in $W^{1,2}\left([0, T] ; \mathrm{H}_{i}\right)$. Indeed, it follows from (3.8) that

$$
(\forall n \in \mathbb{N})\left(\forall z \in \mathcal{H}_{i}\right) \quad J_{\gamma_{n} A_{i}} z \in \operatorname{dom}\left(\gamma_{n} A_{i}\right) \subset \mathcal{W}_{i} \subset W^{1,2}\left([0, T] ; \mathrm{H}_{i}\right) .
$$

As a result, we deduce from (3.19) that $\left(a_{i, n}\right)_{n \in \mathbb{N}}$ lies in $W^{1,2}\left([0, T] ; \mathrm{H}_{i}\right)$. On the other hand, by construction, $\left(y_{i, n}\right)_{n \in \mathbb{N}}$ lies in $\mathcal{W}_{i} \subset W^{1,2}\left([0, T] ; \mathrm{H}_{i}\right)$. In view of (3.6) and (i) in Algorithm 3.2, $\left(x_{i, n}\right)_{n \in \mathbb{N}}$ is therefore in $W^{1,2}\left([0, T] ; \mathrm{H}_{i}\right)$. Next, let us show that $\left(x_{i, n}^{\prime}\right)_{n \in \mathbb{N}}$ is bounded in $L^{2}\left([0, T] ; \mathrm{H}_{i}\right)$. To this end, let $n \in \mathbb{N}$ and set

$$
\left.w_{i, n}(t)=\frac{x_{i, n}(t)-y_{i, n}(t)}{\gamma_{n}}-\mathrm{B}_{i}\left(x_{1, n}(t), \ldots, x_{m, n}(t)\right)-b_{i, n}(t)-y_{i, n}^{\prime}(t)-e_{i, n}(t) \text { a.e. on }\right] 0, T[.
$$

Then we derive from (3.5) that

$$
\left.w_{i, n}(t) \in \partial \mathrm{f}_{i}\left(y_{i, n}(t)\right) \text { a.e. on }\right] 0, T[.
$$

Hence, since $w_{i, n} \in \mathcal{H}_{i}$, it follows from [15, Lemme 3.3] that

$$
\left.\frac{d\left(\mathbf{f}_{i} \circ y_{i, n}\right)(t)}{d t}=\left\langle w_{i, n}(t) \mid y_{i, n}^{\prime}(t)\right\rangle_{\mathrm{H}_{i}} \text { a.e. on }\right] 0, T[.
$$

On the other hand, since $y_{i, n} \in \mathcal{W}_{i}$, we have $y_{i, n}(T)=y_{i, n}(0)$. Therefore

$$
\int_{0}^{T}\left\langle w_{i, n}(t) \mid y_{i, n}^{\prime}(t)\right\rangle_{\mathbf{H}_{i}} d t=\int_{0}^{T} \frac{d\left(\mathrm{f}_{i} \circ y_{i, n}\right)(t)}{d t} d t=\mathrm{f}_{i}\left(y_{i, n}(T)\right)-\mathrm{f}_{i}\left(y_{i, n}(0)\right)=0
$$

and, furthermore,

$$
\int_{0}^{T}\left\langle y_{i, n}(t) \mid y_{i, n}^{\prime}(t)\right\rangle_{\mathbf{H}_{i}} d t=\frac{1}{2} \int_{0}^{T} \frac{d\left\|y_{i, n}(t)\right\|_{\mathbf{H}_{i}}^{2}}{d t} d t=\frac{\left\|y_{i, n}(T)\right\|_{\mathrm{H}_{i}}^{2}-\left\|y_{i, n}(0)\right\|_{\mathbf{H}_{i}}^{2}}{2}=0 .
$$

We deduce from (3.28), (3.25), and (3.29) that

$$
\begin{aligned}
0= & \int_{0}^{T}\left\langle\frac{x_{i, n}(t)}{\gamma_{n}} \mid y_{i, n}^{\prime}(t)\right\rangle_{\mathrm{H}_{i}} d t-\int_{0}^{T}\left\langle\mathrm{~B}_{i}\left(x_{1, n}(t), \ldots, x_{m, n}(t)\right) \mid y_{i, n}^{\prime}(t)\right\rangle_{\mathrm{H}_{i}} d t \\
& -\int_{0}^{T}\left\langle b_{i, n}(t) \mid y_{i, n}^{\prime}(t)\right\rangle_{\mathrm{H}_{i}} d t-\int_{0}^{T}\left\|y_{i, n}^{\prime}(t)\right\|_{\mathrm{H}_{i}}^{2} d t-\int_{0}^{T}\left\langle e_{i, n}(t) \mid y_{i, n}^{\prime}(t)\right\rangle_{\mathrm{H}_{i}} d t .
\end{aligned}
$$

Thus, using Cauchy-Schwarz, the inequality $\gamma_{n} \geq \varepsilon$, and (3.13), we obtain

$$
\left\|y_{i, n}^{\prime}\right\|^{2} \leq\left(\frac{1}{\varepsilon}\left\|x_{i, n}\right\|+\left\|B_{i}\left(x_{1, n}, \ldots, x_{m, n}\right)\right\|+\left\|b_{i, n}\right\|+\left\|e_{i, n}\right\|\right)\left\|y_{i, n}^{\prime}\right\| .
$$

In turn, it follows from (3.6) that

$$
\left\|x_{i, n+1}^{\prime}\right\| \leq \lambda_{i, n}\left\|x_{i, n}^{\prime}\right\|+\left(1-\lambda_{i, n}\right)\left(\frac{1}{\varepsilon}\left\|x_{i, n}\right\|+\left\|B_{i}\left(x_{1, n}, \ldots, x_{m, n}\right)\right\|+\left\|b_{i, n}\right\|+\left\|e_{i, n}\right\|\right) .
$$


On the other hand, arguing as in (3.16), we derive from (3.23) that

$$
\left\|B_{i}\left(x_{1, n}, \ldots, x_{m, n}\right)\right\| \leq \sqrt{2 T\left\|\mathrm{~b}_{i}\right\|_{\mathrm{H}_{i}}^{2}+\frac{2 m \sigma^{2}}{\beta^{2}}} \leq \sqrt{2 T}\left\|\mathrm{~b}_{i}\right\|_{\mathrm{H}_{i}}+\sqrt{2 m} \frac{\sigma}{\beta} .
$$

Hence, using (ii) in Algorithm 3.2, we derive by induction from (3.32) that

$$
\left\|x_{i, n}^{\prime}\right\| \leq \max \left\{\left\|x_{i, 0}^{\prime}\right\|, \frac{\sigma}{\varepsilon}+\sqrt{2 T}\left\|\mathrm{~b}_{i}\right\|_{\mathrm{H}_{i}}+\sqrt{2 m} \frac{\sigma}{\beta}+\sup _{k \in \mathbb{N}}\left(\left\|b_{i, k}\right\|+\left\|e_{i, k}\right\|\right)\right\} .
$$

This shows the boundedness of $\left(x_{i, n}^{\prime}\right)_{n \in \mathbb{N}}$ in $L^{2}\left([0, T] ; \mathrm{H}_{i}\right)$. Now let $z$ be the weak limit in $L^{2}\left([0, T] ; \mathrm{H}_{i}\right)$ of an arbitrary weakly convergent subsequence of $\left(x_{i, n}^{\prime}\right)_{n \in \mathbb{N}}$. Since $\left(x_{i, n}\right)_{n \in \mathbb{N}}$ converges weakly in $L^{2}\left([0, T] ; \mathrm{H}_{i}\right)$ to $x_{i}$, it therefore follows from [48, Proposition 23.19] that $z=x_{i}^{\prime}$. In turn, this shows that $\left(x_{i, n}^{\prime}\right)_{n \in \mathbb{N}}$ converges weakly in $L^{2}\left([0, T] ; \mathrm{H}_{i}\right)$ to $x_{i}^{\prime}$.

\section{The variational case}

We study a special case of Problem 1.1 which yields a variational formulation that extends (1.5).

Recall that, for every $f \in \Gamma_{0}(\mathcal{H})$ and every $x \in \mathcal{H}$, the function $y \mapsto f(y)+\|x-y\|^{2} / 2$ admits a unique minimizer, which is denoted by $\operatorname{prox}_{f} x$. The proximity operator thus defined can be expressed as $\operatorname{prox}_{f}=J_{\partial f}[36]$.

Problem 4.1 Let $\left(\mathcal{H}_{i}\right)_{1 \leq i \leq m}$ and $\left(\mathcal{G}_{k}\right)_{1 \leq k \leq p}$ be real Hilbert spaces. For every $i \in\{1, \ldots, m\}$, let $f_{i} \in \Gamma_{0}\left(\mathcal{H}_{i}\right)$ and, for every $k \in\{1, \ldots, p\}$, let $\left.\tau_{k} \in\right] 0,+\infty\left[\right.$, let $\varphi_{k}: \mathcal{G}_{k} \rightarrow \mathbb{R}$ be a $\tau_{k}$-Lipschitzdifferentiable convex function, and let $L_{k i}: \mathcal{H}_{i} \rightarrow \mathcal{G}_{k}$ be linear and bounded. It is assumed that $\min _{1 \leq k \leq p} \sum_{i=1}^{m}\left\|L_{k i}\right\|^{2}>0$. The problem is to

$$
\underset{x_{1} \in \mathcal{H}_{1}, \ldots, x_{m} \in \mathcal{H}_{m}}{\operatorname{minimize}} \sum_{i=1}^{m} f_{i}\left(x_{i}\right)+\sum_{k=1}^{p} \varphi_{k}\left(\sum_{i=1}^{m} L_{k i} x_{i}\right),
$$

under the assumption that solutions exist.

Algorithm 4.2 Set

$$
\beta=\frac{1}{p \max _{1 \leq k \leq p} \tau_{k} \sum_{i=1}^{m}\left\|L_{k i}\right\|^{2}} .
$$

Fix $\varepsilon \in] 0, \min \{1, \beta\}\left[,\left(\gamma_{n}\right)_{n \in \mathbb{N}}\right.$ in $[\varepsilon, 2 \beta-\varepsilon],\left(\lambda_{n}\right)_{n \in \mathbb{N}}$ in $[0,1-\varepsilon]$, and $\left(x_{i, 0}\right)_{1 \leq i \leq m} \in \mathcal{H}_{1} \times \cdots \times \mathcal{H}_{m}$. Set, for every $n \in \mathbb{N}$,

$$
\left\{\begin{aligned}
x_{1, n+1}= & \lambda_{1, n} x_{1, n}+ \\
& \left(1-\lambda_{1, n}\right)\left(\operatorname{prox}_{\gamma_{n} f_{1, n}}\left(x_{1, n}-\gamma_{n}\left(\sum_{k=1}^{p} L_{k 1}^{*} \nabla \varphi_{k}\left(\sum_{j=1}^{m} L_{k j} x_{j, n}\right)+b_{1, n}\right)\right)+a_{1, n}\right), \\
\vdots & \lambda_{m, n} x_{m, n}+ \\
& \left(1-\lambda_{m, n}\right)\left(\operatorname{prox}_{\gamma_{n} f_{m, n}}\left(x_{m, n}-\gamma_{n}\left(\sum_{k=1}^{p} L_{k m}^{*} \nabla \varphi_{k}\left(\sum_{j=1}^{m} L_{k j} x_{j, n}\right)+b_{m, n}\right)\right)+a_{m, n}\right),
\end{aligned}\right.
$$


where, for every $i \in\{1, \ldots, m\}$, the following hold.

(i) $\left(f_{i, n}\right)_{n \in \mathbb{N}}$ are functions in $\Gamma_{0}\left(\mathcal{H}_{i}\right)$ such that

$$
(\forall \rho \in] 0,+\infty[) \sum_{n \in \mathbb{N}} \sup _{\|y\| \leq \rho}\left\|\operatorname{prox}_{\gamma_{n} f_{i, n}} y-\operatorname{prox}_{\gamma_{n} f_{i}} y\right\|<+\infty .
$$

(ii) $\left(a_{i, n}\right)_{n \in \mathbb{N}}$ and $\left(b_{i, n}\right)_{n \in \mathbb{N}}$ are sequences in $\mathcal{H}_{i}$ such that $\sum_{n \in \mathbb{N}}\left\|a_{i, n}\right\|<+\infty$ and $\sum_{n \in \mathbb{N}}\left\|b_{i, n}\right\|<$ $+\infty$.

(iii) $\left(\lambda_{i, n}\right)_{n \in \mathbb{N}}$ is a sequence in $\left[0,1\left[\right.\right.$ such that $\sum_{n \in \mathbb{N}}\left|\lambda_{i, n}-\lambda_{n}\right|<+\infty$.

We now turn our attention to the asymptotic behavior of Algorithm 4.2 (strong convergence conditions can be derived from Theorem 2.10).

Theorem 4.3 Let $\left(\left(x_{i, n}\right)_{n \in \mathbb{N}}\right)_{1 \leq i \leq m}$ be sequences generated by Algorithm 4.2. Then, for every $i \in\{1, \ldots, m\},\left(x_{i, n}\right)_{n \in \mathbb{N}}$ converges weakly to a point $x_{i} \in \mathcal{H}_{i}$, and $\left(x_{i}\right)_{1 \leq i \leq m}$ is a solution to Problem 4.1.

Proof. Problem 4.1 is a special case of Problem 1.1 where, for every $i \in\{1, \ldots, m\}$,

$$
A_{i}=\partial f_{i} \quad \text { and } \quad B_{i}:\left(x_{j}\right)_{1 \leq j \leq m} \mapsto \sum_{k=1}^{p} L_{k i}^{*} \nabla \varphi_{k}\left(\sum_{j=1}^{m} L_{k j} x_{j}\right) .
$$

Indeed, define $\mathcal{H}$ as in the proof of Theorem 2.9 and set

$$
\boldsymbol{f}: \mathcal{H} \rightarrow]-\infty,+\infty]:\left(x_{i}\right)_{1 \leq i \leq m} \mapsto \sum_{i=1}^{m} f_{i}\left(x_{i}\right)
$$

and

$$
\boldsymbol{g}: \mathcal{H} \rightarrow \mathbb{R}:\left(x_{i}\right)_{1 \leq i \leq m} \mapsto \sum_{k=1}^{p} \varphi_{k}\left(\sum_{i=1}^{m} L_{k i} x_{i}\right) .
$$

Then $\boldsymbol{f}$ and $\boldsymbol{g}$ are in $\Gamma_{0}(\mathcal{H})$ and it follows from Fermat's rule and elementary subdifferential calculus that, for every $\left(x_{1}, \ldots, x_{m}\right) \in \mathcal{H}$,

$$
\begin{aligned}
\left(x_{1}, \ldots, x_{m}\right) \text { solves }(4.1) & \Leftrightarrow \quad(0, \ldots, 0) \in \partial(\boldsymbol{f}+\boldsymbol{g})\left(x_{1}, \ldots, x_{m}\right) \\
& \Leftrightarrow(0, \ldots, 0) \in \partial \boldsymbol{f}\left(x_{1}, \ldots, x_{m}\right)+\nabla \boldsymbol{g}\left(x_{1}, \ldots, x_{m}\right) \\
& \Leftrightarrow \quad(\forall i \in\{1, \ldots, m\}) 0 \in \partial f_{i}\left(x_{i}\right)+\sum_{k=1}^{p} L_{k i}^{*} \nabla \varphi_{k}\left(\sum_{j=1}^{m} L_{k j} x_{j}\right) \\
& \Leftrightarrow(\forall i \in\{1, \ldots, m\}) \quad 0 \in A_{i} x_{i}+B_{i}\left(x_{1}, \ldots, x_{m}\right) .
\end{aligned}
$$

Next, let us show that the family $\left(B_{i}\right)_{1 \leq i \leq m}$ in (4.5) satisfies (1.6) with $\beta$ as in (4.2). First, Lemma 2.6 asserts that, for every $k \in\{1, \ldots, p\}, \nabla \varphi_{k}$ is $\tau_{k}^{-1}$-cocoercive. Hence, for every $i \in$ 
$\{1, \ldots, m\}, x_{i} \in \mathcal{H}_{i}$, and $y_{i} \in \mathcal{H}_{i}$, it follows from (4.5), (4.2), and the convexity of $\|\cdot\|^{2}$ that

$$
\begin{aligned}
& \sum_{i=1}^{m}\left\langle B_{i}\left(x_{1}, \ldots, x_{m}\right)-B_{i}\left(y_{1}, \ldots, y_{m}\right) \mid x_{i}-y_{i}\right\rangle \\
& =\sum_{i=1}^{m} \sum_{k=1}^{p}\left\langle L_{k i}^{*}\left(\nabla \varphi_{k}\left(\sum_{j=1}^{m} L_{k j} x_{j}\right)-\nabla \varphi_{k}\left(\sum_{j=1}^{m} L_{k j} y_{j}\right)\right) \mid x_{i}-y_{i}\right\rangle \\
& =\sum_{i=1}^{m} \sum_{k=1}^{p}\left\langle\nabla \varphi_{k}\left(\sum_{j=1}^{m} L_{k j} x_{j}\right)-\nabla \varphi_{k}\left(\sum_{j=1}^{m} L_{k j} y_{j}\right) \mid L_{k i}\left(x_{i}-y_{i}\right)\right\rangle \\
& =\sum_{k=1}^{p}\left\langle\nabla \varphi_{k}\left(\sum_{j=1}^{m} L_{k j} x_{j}\right)-\nabla \varphi_{k}\left(\sum_{j=1}^{m} L_{k j} y_{j}\right) \mid \sum_{i=1}^{m} L_{k i} x_{i}-\sum_{i=1}^{m} L_{k i} y_{i}\right\rangle \\
& \geq \sum_{k=1}^{p} \frac{1}{\tau_{k}}\left\|\nabla \varphi_{k}\left(\sum_{j=1}^{m} L_{k j} x_{j}\right)-\nabla \varphi_{k}\left(\sum_{j=1}^{m} L_{k j} y_{j}\right)\right\|^{2} \\
& =\sum_{k=1}^{p} \frac{1}{\tau_{k} \sum_{i=1}^{m}\left\|L_{k i}\right\|^{2}} \sum_{i=1}^{m}\left\|L_{k i}\right\|^{2}\left\|\nabla \varphi_{k}\left(\sum_{j=1}^{m} L_{k j} x_{j}\right)-\nabla \varphi_{k}\left(\sum_{j=1}^{m} L_{k j} y_{j}\right)\right\|^{2} \\
& \geq p \beta \sum_{k=1}^{p} \sum_{i=1}^{m}\left\|L_{k i}\right\|^{2}\left\|\nabla \varphi_{k}\left(\sum_{j=1}^{m} L_{k j} x_{j}\right)-\nabla \varphi_{k}\left(\sum_{j=1}^{m} L_{k j} y_{j}\right)\right\|^{2} \\
& \geq \beta \sum_{i=1}^{m} p \sum_{k=1}^{p}\left\|L_{k i}^{*}\left(\nabla \varphi_{k}\left(\sum_{j=1}^{m} L_{k j} x_{j}\right)-\nabla \varphi_{k}\left(\sum_{j=1}^{m} L_{k j} y_{j}\right)\right)\right\|^{2} \\
& \geq \beta \sum_{i=1}^{m}\left\|\sum_{k=1}^{p} L_{k i}^{*} \nabla \varphi_{k}\left(\sum_{j=1}^{m} L_{k j} x_{j}\right)-\sum_{k=1}^{p} L_{k i}^{*} \nabla \varphi_{k}\left(\sum_{j=1}^{m} L_{k j} y_{j}\right)\right\|^{2} .
\end{aligned}
$$

This shows that (1.6) holds. Furthermore, upon setting

$$
(\forall i \in\{1, \ldots, m\})(\forall n \in \mathbb{N}) \quad A_{i, n}=\partial f_{i, n} \quad \text { and } \quad B_{i, n}=B_{i}
$$

we deduce from (4.4) that Algorithm 4.2 is a particular case of Algorithm 2.2. Altogether, Theorem 4.3 follows from Theorem 2.9.

Here are a couple of applications of Problem 4.1.

Example 4.4 (traffic theory) Consider a network with $M$ links indexed by $j \in\{1, \ldots, M\}$ and $N$ paths indexed by $l \in\{1, \ldots, N\}$, linking a subset of $Q$ origin-destination node pairs indexed by $k \in\{1, \ldots, Q\}$. There are $m$ types of users indexed by $i \in\{1, \ldots, m\}$ transiting on the network. For every $i \in\{1, \ldots, m\}$ and $l \in\{1, \ldots, N\}$, let $\xi_{i l} \in \mathbb{R}$ be the flux of user $i$ on path $l$ and let $x_{i}=\left(\xi_{i l}\right)_{1 \leq l \leq N}$ be the flow associated with user $i$. A standard problem in traffic theory is to find a Wardrop equilibrium [45] of the network, i.e., flows $\left(x_{i}\right)_{1 \leq i \leq m}$ such that the costs in all paths actually used are equal and less than those a single user would face on any unused path. Such an equilibrium can be obtained by solving the variational problem [13, 37, 41]

$$
\underset{x_{1} \in C_{1}, \ldots, x_{m} \in C_{m}}{\operatorname{minimize}} \sum_{j=1}^{M} \int_{0}^{h_{j}\left(x_{1}, \ldots, x_{m}\right)} \phi_{j}(h) d h,
$$


where $\phi_{j}: \mathbb{R} \rightarrow[0,+\infty[$ is a strictly increasing $\tau$-Lipschitz continuous function modeling the cost of transiting on link $j$ and $h_{j}\left(x_{1}, \ldots, x_{m}\right)$ is the total flow through link $j$, which can be expressed as $h_{j}\left(x_{1}, \ldots, x_{m}\right)=\sum_{i=1}^{m}\left(L x_{i}\right)^{\top} e_{j}$, where $e_{j}$ is the $j$ th canonical basis vector of $\mathbb{R}^{M}$ and $L$ is an $M \times N$ binary matrix with $j$ lth entry equal to 1 or 0 , according as link $j$ belongs to path $l$ or not. Furthermore, each closed and convex constraint set $C_{i}$ in (4.11) is defined as $C_{i}=$ $\left\{\left(\eta_{l}\right)_{1 \leq l \leq N} \in\left[0,+\infty\left[^{N} \mid(\forall k \in\{1, \ldots, Q\}) \sum_{l \in N_{k}} \eta_{l}=\delta_{i k}\right\}\right.\right.$, where $\varnothing \neq N_{k} \subset\{1, \ldots, N\}$ is the set of paths linking the pair $k$ and $\delta_{i k} \in[0,+\infty$ [ is the flow of user $i$ that must transit from the origin to the destination of pair $k$ (for more details on network flows, see [40,41]). Upon setting

$$
\varphi_{1}: \mathbb{R}^{M} \rightarrow \mathbb{R}:\left(\nu_{j}\right)_{1 \leq j \leq M} \mapsto \sum_{j=1}^{M} \int_{0}^{\nu_{j}} \phi_{j}(h) d h,
$$

problem (4.11) can be written as

$$
\underset{x_{1} \in \mathbb{R}^{N}, \ldots, x_{m} \in \mathbb{R}^{N}}{\operatorname{minimize}} \sum_{i=1}^{m} \iota_{C_{i}}\left(x_{i}\right)+\varphi_{1}\left(\sum_{i=1}^{m} L x_{i}\right) .
$$

Since $\varphi_{1}$ is strictly convex and $\tau$-Lipschitz-differentiable, (4.13) is a particular instance of Problem 4.1 with $p=1, \mathcal{G}_{1}=\mathbb{R}^{M}$ and $(\forall i \in\{1, \ldots, m\}) \mathcal{H}_{i}=\mathbb{R}^{N}, f_{i}=\iota_{C_{i}}$, and $L_{1 i}=L$. Accordingly, Theorem 4.3 asserts that (4.13) can be solved by Algorithm 4.2 which, with the choice of parameters $\left.\gamma_{n} \equiv \gamma \in\right] 0,2 / \tau\left[, \lambda_{i, n} \equiv 0, \lambda_{n} \equiv 0, a_{i, n} \equiv 0\right.$, and $b_{i, n} \equiv 0$, yields

$$
(\forall i \in\{1, \ldots, m\}) \quad x_{i, n+1}=P_{C_{i}}\left(x_{i, n}-\gamma L^{\top}\left(\phi_{1}\left(\rho_{1, n}\right), \ldots, \phi_{M}\left(\rho_{M, n}\right)\right)\right),
$$

where $\left(\rho_{1, n}, \ldots, \rho_{M, n}\right)=\sum_{j=1}^{m} L x_{j, n}$. In the special case when $m=1$ the algorithm described in (4.14) is proposed in [14]. Let us note that, as an alternative to $\varphi_{1}$ in (4.12), we can consider the function

$$
\varphi_{1}: \mathbb{R}^{M} \rightarrow \mathbb{R}:\left(\nu_{j}\right)_{1 \leq j \leq M} \mapsto \sum_{j=1}^{M} \nu_{j} \phi_{j}\left(\nu_{j}\right),
$$

under suitable assumptions on $\left(\phi_{j}\right)_{1 \leq j \leq M}$. In this case, (4.13) reduces to the problem of finding the social optimum in the network [41], that is

$$
\underset{x_{1} \in C_{1}, \ldots, x_{m} \in C_{m}}{\operatorname{minimize}} \sum_{j=1}^{M} h_{j}\left(x_{1}, \ldots, x_{m}\right) \phi_{j}\left(h_{j}\left(x_{1}, \ldots, x_{m}\right)\right),
$$

which can also be solved with Algorithm 4.2.

Example 4.5 (best approximation) The convex feasibility problem is to find a point in the intersection of closed convex subsets $\left(C_{i}\right)_{1 \leq i \leq m}$ of a real Hilbert space $\mathcal{H}[10,21]$. This problem arises in many applications in engineering and the physical sciences [17, 19]. In many instances, the intersection of the sets $\left(C_{i}\right)_{1 \leq i \leq m}$ may turn out to be empty and a relaxation of this problem in the presence of a hard constraint represented by $C_{1}$ is to [23]

$$
\underset{x_{1} \in C_{1}}{\operatorname{minimize}} \frac{1}{2} \sum_{i=2}^{m} \omega_{i} d_{C_{i}}^{2}\left(x_{1}\right)
$$

where $\left(\omega_{i}\right)_{2 \leq i \leq m}$ are strictly positive weights such that $\max _{2 \leq i \leq m} \omega_{i}=1$. We assume that this problem admits at least one solution, as is the case when one of the sets in $\left(C_{i}\right)_{1 \leq i \leq m}$ is bounded 
[23, Proposition 4]. Since, for every $i \in\{2, \ldots, m\}$ and every $x_{1} \in C_{1}, d_{C_{i}}^{2}\left(x_{1}\right)=\min _{x_{i} \in C_{i}}\left\|x_{1}-x_{i}\right\|^{2}$, (4.17) can be reformulated as

$$
\underset{x_{1} \in C_{1}, \ldots, x_{m} \in C_{m}}{\operatorname{minimize}} \frac{1}{2} \sum_{k=1}^{m-1} \omega_{k+1}\left\|x_{1}-x_{k+1}\right\|^{2} .
$$

This is a special instance of Problem 4.1 with $p=m-1$ and, for every $i \in\{1, \ldots, m\}, f_{i}=\iota_{C_{i}}$ and

$$
(\forall k \in\{1, \ldots, m-1\}) \quad \varphi_{k}=\frac{\omega_{k+1}}{2}\|\cdot\|^{2} \quad \text { and } \quad L_{k i}= \begin{cases}\mathrm{Id}, & \text { if } i=1 \\ -\mathrm{Id}, & \text { if } i=k+1 \\ 0, & \text { otherwise }\end{cases}
$$

We can derive from Algorithm 4.2 an algorithm which, by Theorem 4.3, generates orbits that are guaranteed to converge weakly to a solution to (4.18). Indeed, in this case, (4.2) yields $\beta=$ $1 /(2(m-1))$. For example, upon setting $\left.\gamma_{n} \equiv \gamma \in\right] 0,1 /(m-1)\left[, \lambda_{n} \equiv 0, \lambda_{i, n} \equiv 0, a_{i, n} \equiv 0, b_{i, n} \equiv 0\right.$, and $f_{i, n}=\iota_{C_{i}}$ for simplicity, Algorithm 4.2 becomes

$$
\left\{\begin{array}{l}
x_{1, n+1}=P_{C_{1}}\left(\left(1-\gamma \sum_{i=2}^{m} \omega_{i}\right) x_{1, n}+\gamma \sum_{i=2}^{m} \omega_{i} x_{i, n}\right) \\
(\forall i \in\{2, \ldots, m\}) \quad x_{i, n+1}=P_{C_{i}}\left(\gamma \omega_{i} x_{1, n}+\left(1-\gamma \omega_{i}\right) x_{i, n}\right) .
\end{array}\right.
$$

In the particular case when $m=2$ and $\gamma=1 / 2$, then $\omega_{2}=1,(4.18)$ is equivalent to finding a best approximation pair relative to $\left(C_{1}, C_{2}\right)[9,11]$, and (4.20) reduces to

$$
\left\{\begin{array}{l}
x_{1, n+1}=P_{C_{1}}\left(\left(x_{1, n}+x_{2, n}\right) / 2\right) \\
x_{2, n+1}=P_{C_{2}}\left(\left(x_{1, n}+x_{2, n}\right) / 2\right) .
\end{array}\right.
$$

\section{References}

[1] F. Acker and M. A. Prestel, Convergence d'un schéma de minimisation alternée, Ann. Fac. Sci. Toulouse V. Sér. Math., vol. 2, pp. 1-9, 1980.

[2] H. Attouch, Variational Convergence for Functions and Operators, Pitman, Boston, MA, 1984.

[3] H. Attouch, J. Bolte, P. Redont, and A. Soubeyran, Alternating proximal algorithms for weakly coupled convex minimization problems - Applications to dynamical games and PDE's, J. Convex Anal., vol. 15, pp. 485-506, 2008.

[4] H. Attouch, P. Redont, and A. Soubeyran, A new class of alternating proximal minimization algorithms with costs-to-move, SIAM J. Optim., vol. 18, pp. 1061-1081, 2007.

[5] J.-P. Aubin and H. Frankowska, Set-Valued Analysis, Birkhäuser, Boston, MA, 1990.

[6] A. Auslender, Problèmes de Minimax via l'Analyse Convexe et les Inégalités Variationnelles: Théorie et Algorithmes, Lecture Notes in Econom. and Math. Systems, vol. 77, Springer-Verlag, New York, 1972.

[7] J.-B. Baillon and G. Haddad, Quelques propriétés des opérateurs angle-bornés et $n$-cycliquement monotones, Israel J. Math., vol. 26, pp. 137-150, 1977.

[8] H. H. Bauschke and J. M. Borwein, On the convergence of von Neumann's alternating projection algorithm for two sets, Set-Valued Anal., vol. 1, pp. 185-212, 1993. 
[9] H. H. Bauschke and J. M. Borwein, Dykstra's alternating projection algorithm for two sets, J. Approx. Theory, vol. 79, pp. 418-443, 1994.

[10] H. H. Bauschke and J. M. Borwein, On projection algorithms for solving convex feasibility problems, SIAM Rev., vol. 38, pp. 367-426, 1996.

[11] H. H. Bauschke, P. L. Combettes, and D. R. Luke, Finding best approximation pairs relative to two closed convex sets in Hilbert spaces, J. Approx. Theory, vol. 127, pp. 178-192, 2004.

[12] H. H. Bauschke, P. L. Combettes, and S. Reich, The asymptotic behavior of the composition of two resolvents, Nonlinear Anal., vol. 60, pp. 283-301, 2005.

[13] M. Beckmann, C. McGuire, and C. Winsten, Studies in Economics of Transportation, Yale University Press, New Haven, 1956.

[14] D. P. Bertsekas and E. M. Gafni, Projection methods for variational inequalities with application to the traffic assignment problem, Math. Programming Stud., vol. 17, pp. 139-159, 1982.

[15] H. Brézis, Opérateurs Maximaux Monotones et Semi-Groupes de Contractions dans les Espaces de Hilbert, North-Holland/Elsevier, New York, 1973.

[16] H. Brézis and M. Sibony, Méthodes d'approximation et d'itération pour les opérateurs monotones, Arch. Rational Mech. Anal., vol. 28, pp. 59-82, 1967/1968.

[17] Y. Censor and S. A. Zenios, Parallel Optimization: Theory, Algorithms, and Applications, Oxford University Press, New York, 1997.

[18] W. Cheney and A. A. Goldstein, Proximity maps for convex sets, Proc. Amer. Math. Soc., vol. 10, pp. 448-450, 1959.

[19] P. L. Combettes, The foundations of set theoretic estimation, Proc. IEEE, vol. 81, pp. 182-208, 1993.

[20] P. L. Combettes, Construction d'un point fixe commun à une famille de contractions fermes, C.R.Acad. Sci. Paris Sér. I Math., vol. 320, pp. 1385-1390, 1995.

[21] P. L. Combettes, Hilbertian convex feasibility problem: Convergence of projection methods, Appl. Math. Optim., vol. 35, pp. 311-330, 1997.

[22] P. L. Combettes, Solving monotone inclusions via compositions of nonexpansive averaged operators, Optimization, vol. 53, pp. 475-504, 2004.

[23] P. L. Combettes and P. Bondon, Hard-constrained inconsistent signal feasibility problems, IEEE Trans. Signal Process., vol. 47, pp. 2460-2468, 1999.

[24] P. L. Combettes and S. A. Hirstoaga, Approximating curves for nonexpansive and monotone operators, J. Convex Anal., vol. 13, pp. 633-646, 2006.

[25] P. L. Combettes and T. Pennanen, Proximal methods for cohypomonotone operators, SIAM J. Control Optim., vol. 43, pp. 731-742, 2004.

[26] P. L. Combettes and V. R. Wajs, Signal recovery by proximal forward-backward splitting, Multiscale Model. Simul., vol. 4, pp. 1168-1200, 2005.

[27] J. C. Dunn, Convexity, monotonicity, and gradient processes in Hilbert space, J. Math. Anal. Appl., vol. 53, pp. 145-158, 1976.

[28] M. Goldburg and R. J. Marks II, Signal synthesis in the presence of an inconsistent set of constraints, IEEE Trans. Circuits and Systems, vol. 32, pp. 647-663, 1985. 
[29] L. G. Gubin, B. T. Polyak, and E. V. Raik, The method of projections for finding the common point of convex sets, U.S.S.R. Comput. Math. Math. Phys., vol. 7, pp. 1-24, 1967.

[30] A. Haraux, Nonlinear Evolution Equations: Global Behavior of Solutions, Lecture Notes in Math., vol. 841, Springer-Verlag, New York, 1981.

[31] B. He, L.-Z. Liao, and S. Wang, Self-adaptive operator splitting methods for monotone variational inequalities, Numer. Math., vol. 94, pp. 715-737, 2003.

[32] A. Kaplan and R. Tichatschke, Proximal point approach and approximation of variational inequalities, SIAM J. Control Optim., vol. 39, pp. 1136-1159, 2000.

[33] N. Lehdili and B. Lemaire, The barycentric proximal method, Comm. Appl. Nonlinear Anal., vol. 6, pp. 29-47, 1999.

[34] E. S. Levitin and B. T. Polyak, Constrained minimization methods, U.S.S.R. Comput. Math. Math. Phys., vol. 6, pp. 1-50, 1966.

[35] B. Mercier, Topics in Finite Element Solution of Elliptic Problems (Lectures on Mathematics, no. 63), Tata Institute of Fundamental Research, Bombay, 1979.

[36] J.-J. Moreau, Proximité et dualité dans un espace hilbertien, Bull. Soc. Math. France, vol. 93, pp. 273-299, 1965.

[37] M. Patriksson, The Traffic Assignment Problem: Models and Methods, Utrecht, The Netherlands, 1994.

[38] W. V. Petryshyn, Construction of fixed points of demicompact mappings in Hilbert space, J. Math. Anal. Appl., vol. 14, pp. 276-284, 1966.

[39] B. T. Polyak, Introduction to Optimization, Optimization Software Inc., New York, 1987.

[40] R. T. Rockafellar, Network Flows and Monotropic Optimization, Wiley, New York, 1984.

[41] Y. Sheffi, Urban Transportation Networks: Equilibrium Analysis with Mathematical Programming Methods, Prentice-Hall, Englewood Cliffs, NJ, 1985.

[42] R. E. Showalter, Monotone Operators in Banach Space and Nonlinear Partial Differential Equations, Mathematical Surveys and Monographs, vol. 49, Amer. Math. Soc., Providence, RI, 1997.

[43] M. Sibony, Méthodes itératives pour les équations et inéquations aux dérivées partielles non linéaires de type monotone, Calcolo, vol. 7, pp. 65-183, 1970.

[44] S. Simons, From Hahn-Banach to Monotonicity, Lecture Notes in Math. 1693, Springer-Verlag, New York, 2008.

[45] J. G. Wardrop, Some theoretical aspects of road traffic research, Proc. Inst. Civil Eng. II, vol. 1, pp. $325-378,1952$.

[46] C. Zălinescu, Convex Analysis in General Vector Spaces, World Scientific, River Edge, NJ, 2002.

[47] E. Zeidler, Nonlinear Functional Analysis and Its Applications I, Springer-Verlag, New York, 1993.

[48] E. Zeidler, Nonlinear Functional Analysis and Its Applications II, Springer-Verlag, New York, 1990.

[49] D. L. Zhu and P. Marcotte, Co-coercivity and its role in the convergence of iterative schemes for solving variational inequalities, SIAM J. Optim., vol. 6, pp. 714-726, 1996. 